\title{
ARTICLE Dendritic cells license regulatory B cells to produce IL-10 and mediate suppression of antigen-specific CD8 T cells
}

\author{
Joanne Boldison ${ }^{1}$, Larissa Camargo Da Rosa ${ }^{1}$, Joanne Davies ${ }^{1}$, Li Wen ${ }^{2}$ and F. Susan Wong ${ }^{1}$
}

Regulatory B cells (Bregs) suppress and reduce autoimmune pathology. However, given the variety of Breg subsets, the role of Bregs in the pathogenesis of type 1 diabetes is still unclear. Here, we dissect this fundamental mechanism. We show that natural protection from type 1 diabetes in nonobese diabetic (NOD) mice is associated with increased numbers of IL-10-producing B cells, while development of type 1 diabetes in NOD mice occurs in animals with compromised IL-10 production by B cells. However, B cells from diabetic mice regain IL-10 function if activated by the innate immune receptor TLR4 and can suppress insulin-specific CD8 T cells in a dendritic cell (DC)-dependent, IL-10-mediated fashion. Suppression of CD8 T cells is reliant on B-cell contact with DCs. This cell contact results in deactivation of DCs, inducing a tolerogenic state, which in turn can regulate pathogenic CD8 T cells. Our findings emphasize the importance of DC-Breg interactions during the development of type 1 diabetes.

Keywords: Regulatory B cells; type 1 diabetes; IL-10; Dendritic cells

Cellular \& Molecular Immunology (2020) 17:843-855; https://doi.org/10.1038/s41423-019-0324-z

Regulatory B cells (Bregs) suppress immune responses and support immune tolerance. Breg cells mitigate inflammation via the production of cytokines such as IL-10, TGF $\beta$ and IL-35, ${ }^{1-3}$ which modulate the response of pathogenic T cells and aid in the generation of other regulatory lymphocyte populations. Bregs can suppress various autoimmune diseases, including models of experimental autoimmune encephalomyelitis (EAE) and arthritis. ${ }^{4-6}$ However, in type 1 diabetes, a dual role for $B$ cells is evident. $B$ cells play a pathological role, as B-cell depletion delays disease onset and protects against diabetes. ${ }^{7-9}$ Conversely, Bregs can prevent the onset of disease in a NOD mouse model. ${ }^{2,10}$ B cells stimulated with lipopolysaccharide (LPS) upregulate FasL expression, increase TGF $\beta$ secretion, and prevent the onset of diabetes upon adoptive transfer. ${ }^{2}$ Furthermore, LPS-stimulated B cells induce CD8 T-cell anergy in a membrane-bound TGF $\beta$-dependent manner. ${ }^{11}$ Activation of $B$ cells through the B-cell receptor (BCR) can also mediate protection from diabetes in NOD mice via an IL10-dependent mechanism. ${ }^{12}$ In patients with type 1 diabetes, IL10-producing B-cell numbers are diminished in the peripheral blood compared with healthy control individuals and autoantibody-positive relatives. ${ }^{13}$

Similar to humans, NOD mice spontaneously develop type 1 diabetes. However, in most, if not all, NOD mouse colonies worldwide, $\sim 20 \%$ (or more) of the NOD mice remain normoglycemic and "protected" from diabetes despite their genetic predisposition. ${ }^{14}$ However, few studies have been performed to uncover the mechanism underlying this natural protection. It is suggested that B cells, specifically anergic $\mathrm{CD} 40^{+} \mathrm{IL}-10$-producing $B$ cells found in the pancreatic islets of long-term normoglycemic (protected) mice, ${ }^{13}$ may confer this natural protection. However, given the heterogeneity among regulatory $\mathrm{B}$ cells, ${ }^{15}$ which can be induced via stimulation with either TLR agonists or an anti-CD40 antibody, we suggest that the mechanism by which $B$ cells induce natural protection still remains undefined.

Here, we report that B cells from NOD mice that have developed diabetes have lost the capacity to produce IL-10, whereas $B$ cells from naturally protected NOD animals have an enhanced frequency of IL-10-producing $B$ cells, regardless of the mode of B-cell activation. However, if $B$ cells from diabetic NOD mice receive signaling through the innate immune receptor TLR4, they regain the IL-10-producing function and are able to suppress insulin-specific CD8 T cells. For B cells to exert this suppression, contact with dendritic cells (DCs) is required and is mediated via IL-10. Direct B-cell-DC interaction deactivates mature conventional DCs and promotes an IL-10-dependent tolerogenic DC population.

\section{RESULTS}

Altered B-cell functions in NOD mice with type 1 diabetes To determine the features of B cells in NOD mice that are "naturally protected" (have not developed diabetes by 30 weeks of age and hereafter referred to as protected, as these mice rarely develop diabetes after 30 weeks), we compared B-cell responses with various stimuli between protected and diabetic NOD mice. B cells were stimulated through adaptive immune costimulatory pathways (anti-CD40 or $\mathrm{B}_{\mathrm{CD} 40}$ ) and through innate immune pathways by microbial products (LPS-B $B_{\text {LPS }}$ or $\left.C p G-B_{C P G}\right){ }^{16-18}$ Unstimulated $B$ cells $\left(B_{U S}\right)$ were used as controls (Fig. 1). All stimuli upregulated the expression of B-cell activation markers, although the upregulation of MHC I and II expression was not statistically different from the expression in $\mathrm{B}_{\mathrm{US}}$. Key differences in

\footnotetext{
${ }^{1}$ Division of Infection and Immunity, Cardiff University School of Medicine, Cardiff CF14 4XN, UK and ${ }^{2}$ Section of Endocrinology, School of Medicine, Yale University, Yale, CT 06520, USA

Correspondence: F. Susan Wong (WongFS@Cardiff.ac.uk)
} 
a
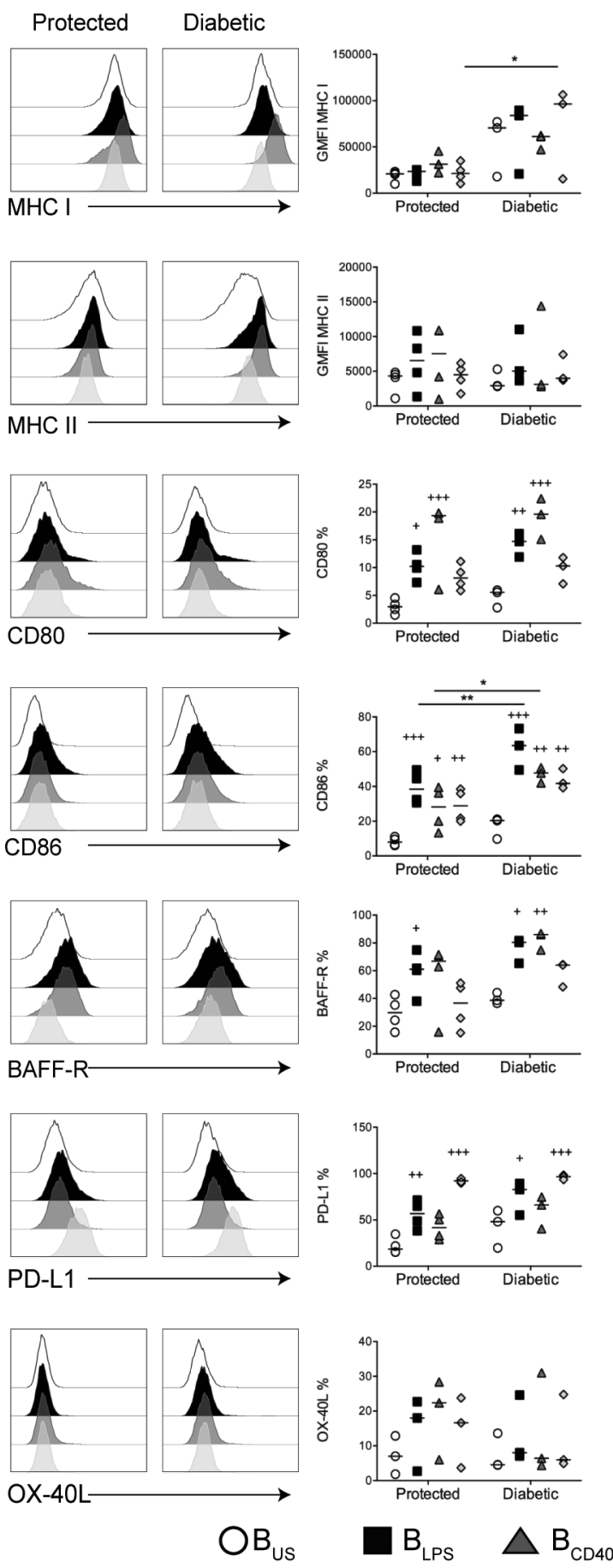

b

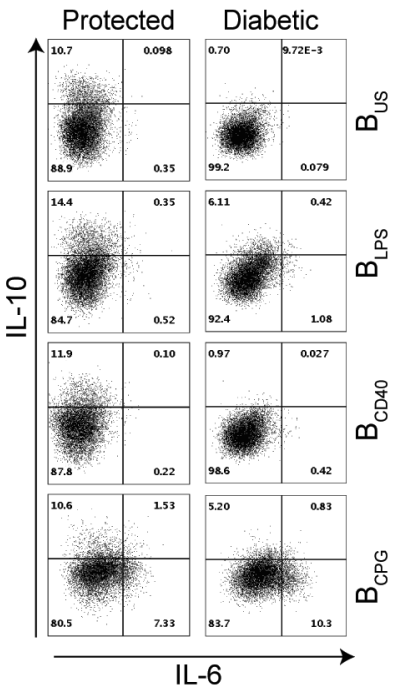

C
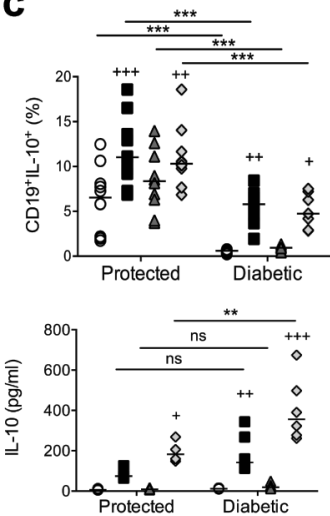

d
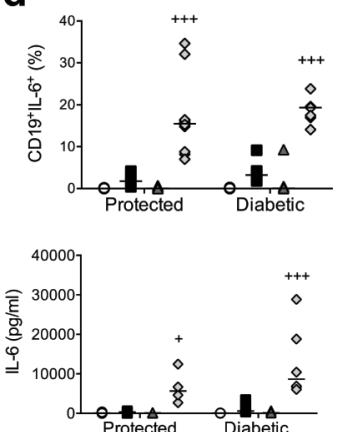

$B_{C P G}$

Fig. 1 Phenotypic analysis of B cells. Splenic B cells from protected ( $>35$ weeks old) and diabetic NOD mice were left unstimulated ( $B_{u s}$; white circle) or stimulated with LPS ( $B_{\mathrm{LPs}}$; black square), anti-CD40 ( $\mathrm{B}_{\mathrm{aCD} 40}$; dark gray triangle) or CPG ( $\mathrm{B}_{\mathrm{CPG}}$; light gray diamond) for $24 \mathrm{~h}$ before being analyzed for surface markers and cytokine production. a Isolated B cells analyzed for various surface markers. Representative flow cytometry plots (left) and a graphical summary (right). b Representative flow cytometry plots for intracytoplasmic IL-6 and IL-10 staining in total splenic B cells. c Graphical summary of intracytoplasmic (top) and secreted (bottom) IL-10. d Graphical summary of intracytoplasmic (top) and secreted (bottom) IL-6. Cytokines in supernatants were measured by MSD. The black line represents the median value. B cells were gated on live CD19 ${ }^{+}$ cells. Data represent at least four independent experiments. ns nonsignificant, ${ }^{+} P<0.05,{ }^{++} P<0.01$, and ${ }^{+++} P<0.001$ versus $\mathrm{B}_{\mathrm{US}}$ (two-way ANOVA). ${ }^{*} P<0.05,{ }^{* *} P<0.01$, and ${ }^{* * *} P<0.001$, protected versus diabetic (two-way ANOVA)

surface markers, between the B cells from protected NOD mice and those from diabetic NOD mice, were that the splenic B cells isolated from protected NOD mice had lower expression of $\mathrm{MHC}$ I and a significantly lower frequency of CD86-expressing B cells, compared with the splenic B cells from diabetic mice (Fig. 1a). In B cells from both protected and diabetic NOD mice, the upregulation of CD80 expression was evident, particularly after anti-CD40 stimulation, compared with $\mathrm{B}_{\mathrm{US}}(p<0.001)$, whereas we observed 
that the upregulation of CD86 expression was greatest if the cells were stimulated by LPS $(p<0.001)$. Interestingly, the innate immune stimuli LPS (TLR4) and CPG (TLR9) resulted in increased upregulation of PD-L1 expression on B cells $(p<0.05$ and $p<$ 0.001 , respectively) compared with $B_{U S}$. We observed that after stimulation with anti-CD40 $\left(\mathrm{B}_{\mathrm{CD} 40}\right)$, a classic B-cell stimulator that functions via an adaptive immune pathway, PD-L1 expression was similar between the stimulated B cells and unstimulated control cells (Fig. 1a). However, no difference in PD-L1 expression was found between protected and diabetic NOD mice, corroborating previous literature. $^{19}$

Next, we determined the cytokine production by B cells activated by the different types of stimuli. First, intracytoplasmic staining revealed significant induction of IL-10 in splenic B cells from both protected and diabetic NOD mice compared with $B_{U S}$ following activation with the innate immune stimuli LPS and CPG $(p<0.05)$, but not following stimulation with anti-CD40, the adaptive immune stimulator (Fig. 1b, c). However, a much higher proportion of B cells from protected NOD mice compared with diabetic NOD mice expressed intracellular IL-10, even without stimulation ( $B_{U S}$ ) ex vivo (Fig. 1c, top graph). In particular, there were considerably lower proportions of IL-10-expressing B cells from diabetic NOD mice upon stimulation with anti-CD40 (Fig. 1C; top graph). B cell secretion of IL-10 was only observed with LPS and CPG stimulation, but not with anti-CD40 stimulation (Fig. 1C; bottom graph), corroborating previous literature. ${ }^{16}$ Surprisingly, B cells from diabetic NOD mice secreted significantly more IL-10, although only when stimulated with CPG, compared with B cells from protected NOD animals (Fig. 1c, bottom graph; $p<0.01$ ). IL10 secretion by LPS-stimulated $B$ cells was modestly increased in diabetic NOD mice compared with protected NOD mice, although this difference was not statistically significant (ns). To address whether the greater proportion of IL-10-producing B cells in protected mice was age related, we investigated $\mathrm{B}^{97}$ congenic mice, which have the same MHC haplotype but are not prone to diabetes, to overcome the technical challenge of there being no NOD mice that develop diabetes beyond 30 weeks of age (Supplementary Fig. 1). $\mathrm{B}^{\mathrm{g}^{7}}$ mice aged $>35$ weeks old exhibited little IL-10 production, indicating that the potential for IL-10 production was unlikely to be age associated.

Only CPG stimulation induced IL-6-producing B cells from both protected and diabetic mice (Fig. 1d), and no significant difference was observed between the protected and diabetic NOD mice for either intracytoplasmic or secreted IL-6 (Fig. 1d), indicating that diabetes protection was not related to a lack of proinflammatory cytokines in B cells.

These data demonstrated that B cells from diabetic NOD mice were more activated than those from mice protected from diabetes, particularly in regard to CD86 expression (Fig. 1a, fourth row). Furthermore, B cells from diabetic mice showed a loss of overall intracellular IL-10 production, but could regain this function if stimulated with TLR4 or TLR9, as highlighted by the IL-10 secretion results. Strikingly, when B cells were stimulated with anti-CD40, only protected mice had the potential to produce cytoplasmic IL-10.

LPS-stimulated B cells suppress antigen-specific CD8 T cells in the presence of DCs

We next investigated whether $B$ cells from diabetic or protected NOD mice could regulate antigen-specific CD8 T cells via both innate and adaptive immune signaling. $B$ cells were cocultured with insulin-specific CD8 T cells from TCR transgenic $\mathrm{G} 9 \mathrm{Ca}^{-/-}$ mice $^{20}$ in the presence of BM-DCs from NOD.PI2tg mice, which express proinsulin (driven by the MHC class II promoter) in antigen-presenting cells (experimental scheme shown in Fig. 2a). This culture system allowed intrinsic antigen-specific presentation, by proinsulin-expressing DCs to insulin-specific CD8 T cells, without exogenous antigen. Considering that NOD.PI2tg DCs express proinsulin, they should be targeted by CD8 T cells from $\mathrm{G} 9 \mathrm{Ca}^{-/-}$mice. Indeed, DC viability was significantly diminished in control CD8 and DC cultures (without B cells) (Fig. 2b). The addition of $B$ cells prevented the killing of DCs by CD8 T cells (Fig. 2b), and importantly, stimulated $B$ cells ( $B_{L P S}, B_{C D 40}$ and $\left.B_{C P G}\right)$ promoted enhanced $D C$ survival compared with unstimulated $B$ cells ( $\left.B_{\cup S}\right)$ (Fig. 2b). Next, we examined the proliferative function of CD8 T cells in response to the intrinsic antigen presented by DCs in a CFSE dilution assay, in the presence of stimulated or unstimulated $B$ cells, from protected and diabetic animals (Fig. 2c-e). CD8 T-cell proliferation was significantly suppressed in the presence of LPS-stimulated $B$ cells $\left(B_{L P S}\right)$ compared with unstimulated $\mathrm{B}$ cells ( $\left.\mathrm{B}_{\text {US }}\right)(p<0.05$; Fig. $2 \mathrm{c}-\mathrm{e})$. Interestingly, no suppression by $B$ cells from either protected or diabetic NOD mice was observed when the $B$ cells were activated by other stimuli (Fig. 2c-e). Control CD8 T-cell proliferation (CD8 + DC alone) is shown in Supplementary Fig. 2a. Similarly, we observed reduced expression of CD44 (Fig. $2 f$, g) and MIP1 $\beta$, the early chemokine produced by activated CD8 T cells, in culture supernatants in the presence of $B_{\text {LPS }}$ (Fig. 2h). To confirm that CD8 T-cell proliferation was impaired in $B_{\text {LPS }}$ cultures and that this change was not due to increased CD8 T-cell death, we assessed CD8 T-cell viability and determined that there was no significant difference between cultures (Supplementary Fig. 2b).

To verify that the suppression mediated by $B_{\text {LPS }}$ was TLR4 dependent, we used $B_{\text {LPS }}$ from TLR4 ${ }^{-/-}$mice in the culture system, and we observed no suppression of CD8 T-cell proliferation or activation (Fig. 2i), confirming that the LPS-mediated suppression was TLR4 dependent. To probe the direct effect of LPS-stimulated $B$ cells on CD8 $T$ cells, we stimulated $\mathrm{G} 9 \mathrm{Ca}^{-1-} \mathrm{CD} 8 \mathrm{~T}$ cells with plate-bound anti-CD3/28 antibodies and assessed CD8 T-cell proliferation in the presence of $B$ cells with or without stimulation. In contrast to DC-stimulated T cells (Fig. 2d), anti-CD3/28 antibodystimulated CD8 T cells cultured with LPS-stimulated B cells ( $B_{\text {LPS }}$ ) did not appear to be suppressed (Fig. 2j). Taken together, our results suggest that the regulation of antigen-specific CD8 $T$ cells by LPS-activated $B$ cells requires the presence of DCs, and importantly, B cells from diabetic mice also have the ability to impair CD8 T-cell responses, if the B cells are stimulated via TLR4.

IL-10 mediates CD8 T-cell suppression

To investigate the IL-10 contribution to CD8 T-cell suppression mediated by LPS-stimulated B cells, we measured the secreted IL10 levels in culture supernatants of the experiments included in Fig. 2. $B_{\text {LPS }}$ from both protected and diabetic mice had significantly increased IL-10 secretion compared with $B_{U S}$ (Fig. 3a). Interestingly, when LPS-stimulated $\mathrm{B}$ cells from aged $\mathrm{B} 6^{\mathrm{g}}{ }^{7}$ transgenic mice ( $>35$ weeks old) were used in our in vitro culture system, we neither observed significant CD8 T-cell suppression (Supplementary Fig. 3a) nor found significant IL-10 production (Supplementary Fig. 3b), demonstrating that IL-10 mediates suppression. Furthermore, the addition of LPS-stimulated NOD B cells had no obvious effect on the secretion of IL- 6 (Supplementary Fig. 3c), suggesting that reduced levels of IL-6 in protected NOD mice were not responsible for the suppression of CD8 T-cell proliferation.

To identify the contribution of IL-10 produced by DCs in our culture system, we analyzed the secreted IL-10 levels in cultures with or without DCs (Fig. 3b). We found a significant increase in the amount of IL-10 secreted when B cells were added to DC+ CD8 T-cell cultures, regardless of the B-cell source and whether the added $B$ cells were unstimulated or stimulated. However, the greatest amount of secreted IL-10 was found in $B_{\text {LPS }}$ cultures. Cultures of CD8 T cells-DCs alone were found to have minimal secreted IL-10 (average $124 \pm 25.4 \mathrm{pg} / \mathrm{ml}$ ), suggesting that upon the addition of B cells, either the DCs or B cells, or both, contributed to the augmented $\mathrm{IL}-10$ production. To further address this finding, $B$ cells ( $B_{U S}, B_{L P S}$ and $B_{C D 40}$ ) from protected, 
$24 \mathrm{hrs}$

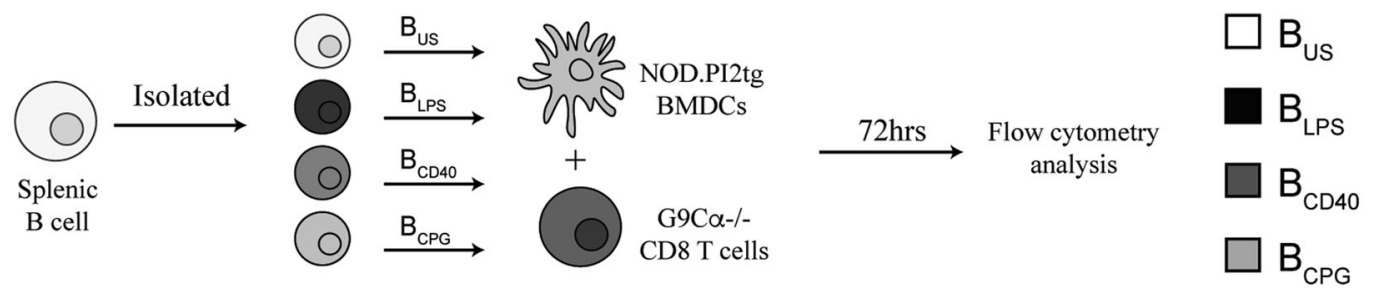

b
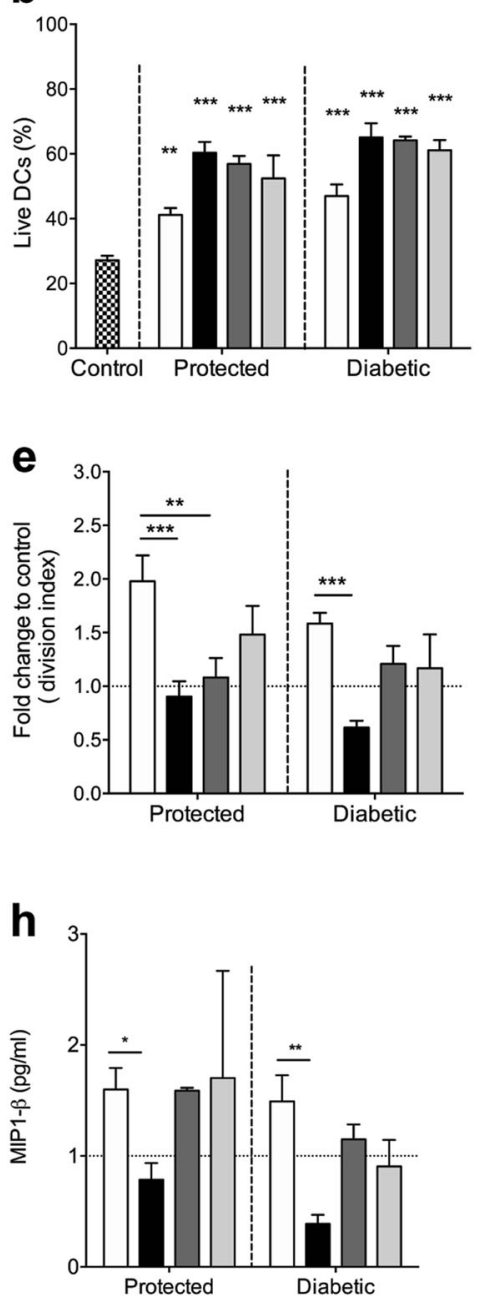

C

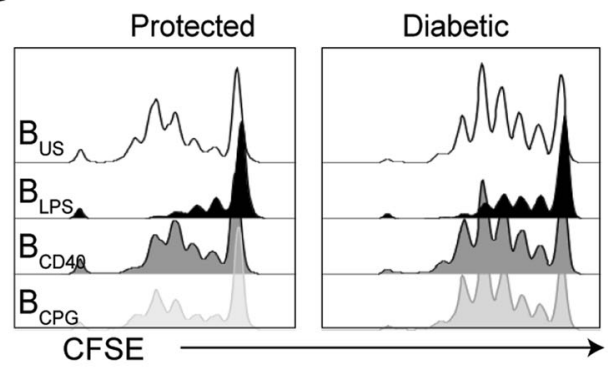

f

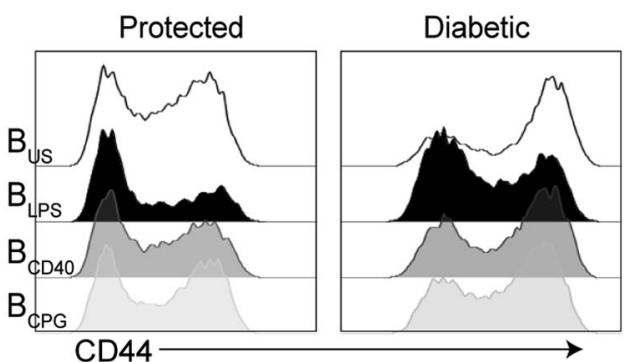

i

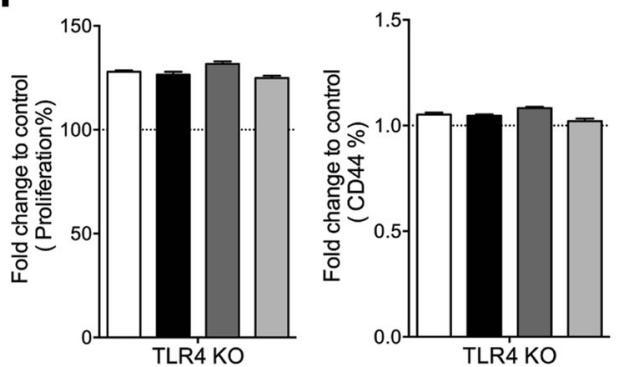

d

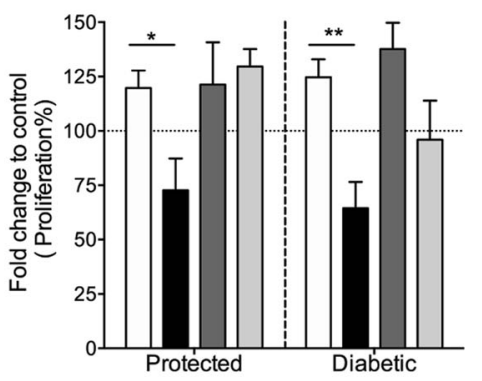

g

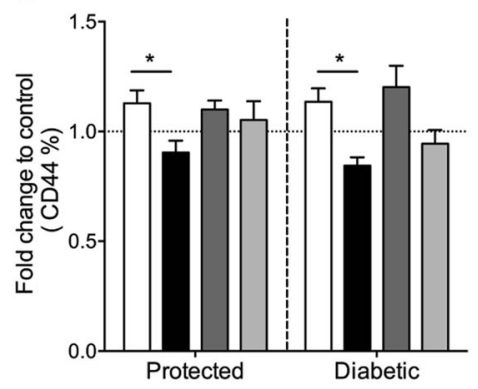

j

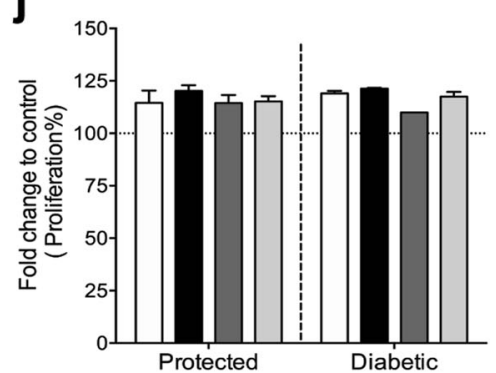

Fig. 2 LPS-stimulated B cells suppress insulin-specific CD8 T cells. NOD.PI2 ${ }^{\text {tg }}$ BM-DCs, CFSE-labeled G9C $\alpha^{-/-}$CD8 T cells, and protected or diabetic NOD mouse-derived splenic $B$ cells that were left unstimulated ( $B_{U S}$, white bar) or stimulated with LPS (B $B_{L P S}$, black bar), anti-CD40 $\left(\mathrm{B}_{\mathrm{aCD} 40}\right.$, dark gray bar), or $\mathrm{CPG}\left(\mathrm{B}_{\mathrm{CPG}}\right.$, light gray bar) were cocultured for 3 days before being examined by flow cytometry. $C D 8 \mathrm{~T}$ cells were gated on $C D 11 c^{-} C D 11 b^{-} C D 19^{-} C D 8^{+}$cells. a Coculture setup and experimental design. $\mathbf{b}$ Live DC percentages $\left(C D 11 c^{+} C D 11 b^{+}\right), * * P<0.01$ and ${ }^{* * *} P<0.001$ versus control (DC $+C D 8$ alone, patterned bar) (one-way ANOVA). c-e CD8 T cell proliferation in B cell cocultures; $\mathbf{c}$ representative CFSE flow plots, $\mathbf{d}$ a graphical representation, and e the division index. $\mathbf{f}$, $\mathbf{g}$ CD44 surface staining of CD8 $T$ cells. $\mathbf{f}$ Representative flow plots and $\mathbf{g}$ a graphical summary. $\mathbf{h}$ ELISA data for the MIP1 $\beta$ cytokine levels in supernatants of cultures. $\mathbf{i}$ NOD.PI2 ${ }^{\text {tg }}$ BM-DCs and G9C $\alpha^{-/-}$CD8 T cells cocultured with splenic B cells from TLR4KO mice, (left) CD8 T-cell proliferation evaluated by CFSE dilution, and (right) CD44 surface staining of CD8 T cells. In $d-i$, data were normalized to control data (DC + CD8 alone). $\mathbf{j}$ G9C $\alpha^{-/-}$CD8 T cell proliferation induced by plate-bound anti-CD3/anti-CD28 antibodies $(0.1$ and $1 \mu \mathrm{g} / \mathrm{ml}$, respectively) in cocultures with unstimulated or stimulated B cells, from protected or diabetic NOD mice. The data are shown as the mean \pm SEM and represent at least three independent experiments. ${ }^{*} P<0.05,{ }^{*} P<0.01$, and ${ }^{* * *} P<0.001$ versus $B_{U S}$ (two-way ANOVA)

diabetic, and IL-10KO NOD mice were cultured with either BM-DCs from NOD.PI2tg mice or BM-DCs from IL-10KO NOD mice (Fig. 3c). IL-10 production in B-cell + DC cultures was dependent on the $B$ cells being capable of producing IL-10, as only a small amount of IL-10 was observed when IL-10KO B cells were cultured with NOD.PI2tg BM-DCs (Fig. 3c). Analysis of the reduced amount of IL-10 produced in the IL-10KO cultures revealed no statistically significant difference between NOD.PI2tg BM-DCs cultured without B cells and those cultured with IL-10KO $B$ cells (Supplementary Fig. 4a). Furthermore, equivalent amounts 
a

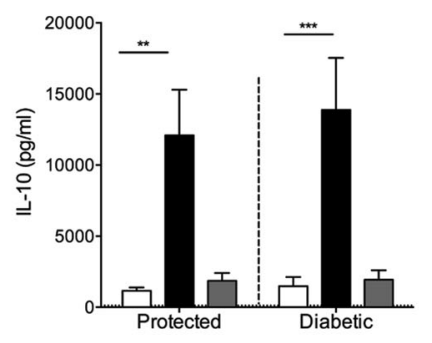

b
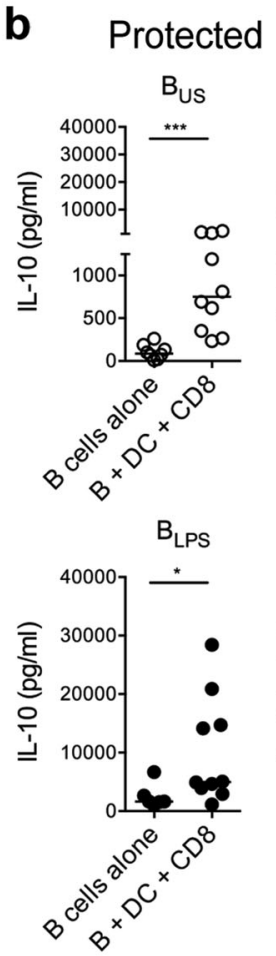

$\mathrm{B}_{\mathrm{aCD} 40}$

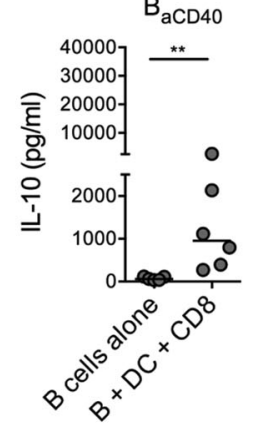

C

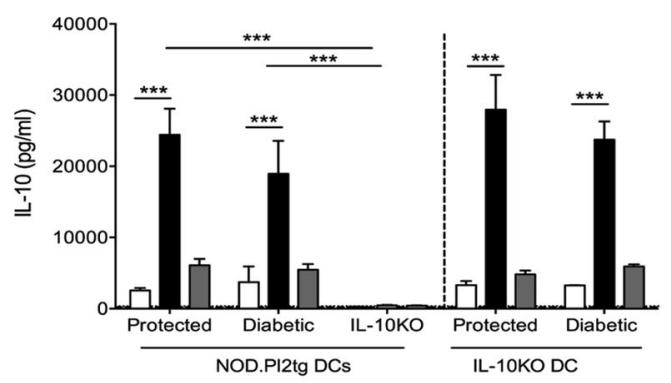

d

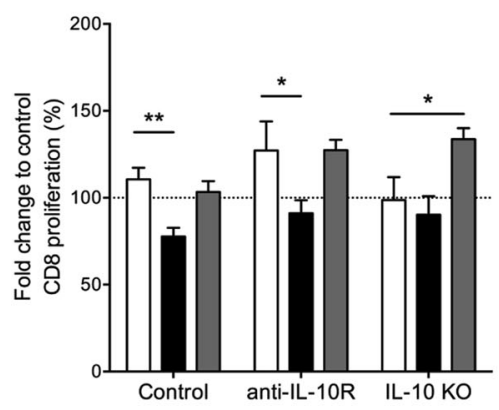

e

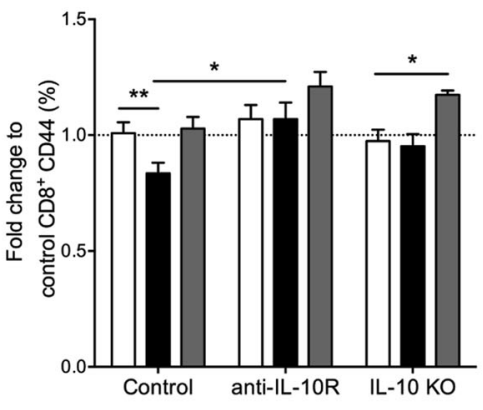

$\mathbf{f}$

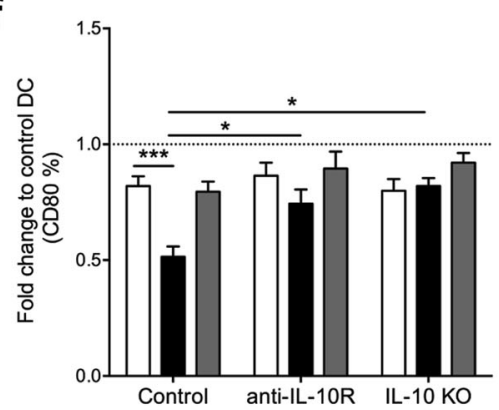

$\mathrm{B}_{\mathrm{CD} 40}$

Fig. 3 IL-10 partially mediates insulin-specific CD8 T-cell suppression. $\mathbf{a}$, b NOD.PI2 ${ }^{\text {tg }}$ BM-DCs, G9C $\alpha^{-/-}$CD8 T cells, and protected or diabetic NOD mouse-derived $B$ cells that were left unstimulated $\left(B_{U S}\right)$ or stimulated with LPS ( $\left.B_{L P S}\right)$ or anti-CD40 ( $B_{\text {aCD }}$ ) were cocultured for 3 days. a IL-10 cytokine levels in B-cell cultures. The dotted line represents the baseline level in a CD8 + DC control culture $(124 \pm 25.4 \mathrm{pg} / \mathrm{ml})$. ${ }^{* *} P<0.01$ and ${ }^{* * *} P$ $<0.001$ (two-way ANOVA). b IL-10 cytokine levels in protected or diabetic NOD B-cell cultures, in which the B cells were plated alone or with NOD. $\mathrm{PI} 2^{\text {tg }} \mathrm{DCs}$ and $\mathrm{G} 9 \mathrm{C} \alpha^{-/-} \mathrm{CD} 8 \mathrm{~T}$ cells $(\mathrm{B}+\mathrm{DC}+\mathrm{CD} 8) .{ }^{*} P<0.05,{ }^{*} P<0.01$, and ${ }^{* * *} P<0.001$ (Mann-Whitney $U$ test); the horizontal line represents the median value. c Unstimulated ( $\left.B_{U S}\right)$ or LPS- $\left(B_{L P S}\right)$ or anti-CD40-stimulated B cells $\left(B_{a C D} 40\right)$ from protected, diabetic, or IL-10KO NOD mice cocultured with BM-DCs from either NOD.PI2 ${ }^{\mathrm{tg}}$ or IL-10KO mice for 3 days before the IL-10 level was measured. The dotted line (NOD.PI2tg) and dashed line (IL-10KO) represent the baseline levels in DC-alone cultures $\left(347 \pm 34.6\right.$ and $218.2 \pm 69.2 \mathrm{pg} / \mathrm{ml}$, respectively). d-f NOD.PI2 ${ }^{\text {tg }}$ BM-DCs and G9C $\alpha^{-/-}$ CD8 T cells cultured with unstimulated $B$ cells $\left(B_{U S}\right)$, LPS- $\left(B_{L P S}\right)$, or anti-CD40-stimulated $B$ cells $\left(B_{a C D}\right.$ ) from protected or diabetic NOD mice treated with either an isotype control (control) or an anti-IL-10 receptor antibody (anti-IL-10R), or IL-10KO B cells. d CD8 T-cell proliferation, e CD44 expression on CD8 T cells, and $\mathbf{f}$ CD80 expression on NOD.PI2 ${ }^{\text {tg }} \mathrm{DCs}$. Data were normalized to control data (DC + CD8 alone, dotted line). ${ }^{*} P<0.05$, ${ }^{*} P<0.01$, and ${ }^{* *} P<0.001$ (two-way ANOVA). The data are shown as the mean \pm SEM. The data shown represent at least three independent experiments 
of IL-10 were produced when B cells from protected or diabetic NOD mice were cultured with IL-10KO BM-DCs (Fig. 3c), indicating that the IL-10 augmentation observed upon the addition of B cells is independent of IL-10 produced by BM-DCs.

As the secreted IL-10 contributed to the suppression of CD8 Tcell proliferation, we evaluated the effect of recombinant IL-10. Surprisingly, increasing concentrations of recombinant IL-10 alone had little effect on $\mathrm{G} \mathrm{Ca}^{-1-} \mathrm{CD} 8$ T-cell proliferation when the T cells were cultured with NOD.PI2tg DCs (Supplementary Fig. 4b). Moreover, blocking the IL-10 receptor (anti-IL-10R antibody) also had little effect on either CD8 T-cell proliferation (Supplementary Fig. 4c, left) or CD44 expression in CD8 T cells (Supplementary Fig. $4 c$, right). Yet, when $B_{\text {LPS }}$ cells were present in the CD8-DC culture system, adding anti-IL-10R antibody not only reduced the suppression of CD8 T-cell proliferation (Fig. 3d) but also promoted a significant increase in CD44 surface expression on CD8 T cells (Fig. 3e), compared to control. This finding was confirmed by using $B$ cells from IL-10KO mice (Fig. 3d, e). In keeping with published reports, ${ }^{21,22} \mathrm{IL}-10$ reduced the expression of costimulatory molecules on DCs to exert immunosuppression (Supplementary Fig. 4d). We also found that the significant reduction in CD80 expression on mature DCs in $B_{\text {LPS }}$ cultures was reversed by the addition of the anti-IL-10R antibody or the use of B cells from IL$10 \mathrm{KO}$ mice (Fig. 3f). However, CD86 expression on DCs was less affected by the addition of the anti-IL-10R antibody or the use of IL-10KO B cells (Supplementary Fig. 4e). Thus far, our results indicated that IL-10 produced by LPS-stimulated B cells downregulated CD80 expression on DCs and suppressed antigenspecific CD8 T cells.

Insulin-specific CD8 T-cell suppression is DC-B-cell contact dependent

To determine if cell contact is also required for $\mathrm{B}_{\mathrm{LPS}}$-mediated CD8 Tcell suppression, we used a transwell culture system. CD8-DC cultures were either in contact with B cells (cont) or separated (trans) from $B$ cells, which were unstimulated or stimulated with LPS ( $\left.B_{\text {LPS }}\right)$ or anti-CD40 $\left(B_{C D 40}\right)$ (Fig. 4a). Our results revealed that $\mathrm{G} \mathrm{Ca}^{-1-} \mathrm{CD} 8$ $\mathrm{T}$-cell proliferation and the expression of CD44 on CD8 T cells were significantly suppressed when $B_{\text {LPS }}$ from either protected or diabetic NOD mice were in contact with the CD8 T cells and DCs. This suppression was significantly reduced when the B cells were separated from the CD8 T-cell-DC cultures (Trans) (Fig. 4a, b).

B-cell-DC contact synergises with cytokine production

Since we observed that CD8 T-cell suppression was mediated via both IL-10- and contact-dependent mechanisms, we sought to determine whether cytokine production was dependent on B cell-DC contact. We measured cytokine secretion when B cells were either in contact with mature NOD.PI2tg BM-DCs or cultured in transwells, in the presence (Fig. $4 c$, e) or absence of $T$ cells (Fig. 4d, f). First, we found that IFN $\gamma$ production was unchanged by the addition of $B$ cells from either protected or diabetic NOD mice, when B cells were not in direct contact (Trans) with CD8 T cells and DCs compared with when B cells were in contact (Cont) (Fig. 4c). However, in the absence of CD8 T cells, B cells from diabetic NOD mice, in direct contact with DCs, induced more IFN $y$ production than B cells from protected NOD mice $(p<0.01)$, regardless of the type of stimulus (Fig. 4d). Furthermore, IFNy production was significantly increased when $B$ cells were stimulated with anti-CD40 $\left(\mathrm{B}_{\mathrm{CD} 40}\right)$ (protected, $p<0.05$, diabetic, $p<0.001$ ), and this effect was also contact dependent (Fig. $4 d$ ).

Second, we found increased IL-10 levels in both protected and diabetic $B_{\text {LPS }}$ cultures in the presence or absence of CD8 T cells, whereas we observed considerably less IL-10 expression in $B_{\text {LPS }}$ transwell cultures (Fig. 4e, f). Interestingly, we observed that IL-10 production in $\mathrm{B}_{\text {LPS }}$ cultures was greater in cultures of cells from diabetic NOD mice, than in those of cells from protected NOD mice, in the presence of CD8 T cells (Fig. 4e). In contrast, in the absence of CD8 T cells, $B_{\text {LPS }}$ cultures of cells from protected NOD mice had the greatest levels of IL-10 (Fig. 4f). Notably, we observed no differences in IL-12p70 or IL- 6 production when comparing B-cell contact and transwell cultures, indicating that these cytokines were not affected by direct B-cell contact (data not shown). Taken together, our data suggest that CD8 T-cell suppression is mediated via IL-10 secretion, which is dependent on direct contact between LPS-stimulated B cells and mature BM-DCs.

\section{$\mathrm{B}_{\mathrm{LPS}}$ condition mature BM-DCs}

We next investigated whether $B$ cells induce tolerance in mature BM-DCs. First, we cocultured B cells with BM-DCs from NOD.PI2tg mice for 3 days. The $B$ cells were then removed, and the conditioned BM-DCs were washed and cocultured with $\mathrm{G} 9 \mathrm{Ca}^{-/-}$ CD8 T cells for 3 days (scheme shown in Fig. 5a), followed by assessment of CD8 T-cell proliferation (Fig. 5b) and activation (Fig. 5c), as well as cytokine production in the DC-CD8 T-cell cultures (Fig. 5d). Overall, CD8 T-cell proliferation (CFSE dilution) and activation (CD44 surface expression) were reduced when mature NOD.PI2tg BM-DCs were exposed to B cells, regardless of the stimulation; however, only $B_{L P S}$, from either protected or diabetic NOD mice significantly suppressed insulin-specific CD8 $\mathrm{T}$ cells compared to the control(no B-cell exposure) (Fig. 5b). Following pre-exposure to B cells stimulated with LPS or antiCD40, the BM-DCs in DC-CD8 T-cell cultures secreted IL-10 (Fig. 5d, left). However, only LPS-stimulated B cells reduced the secretion of proinflammatory cytokines, particularly IL-12p70 (Fig. $5 \mathrm{~d}$, middle) and IL-6 (Fig. $5 \mathrm{~d}$, right), by mature BM-DCs.

$B$ cells modulate the activation of mature conventional DCs We have shown, thus far, that LPS-stimulated B cells suppress CD8 T-cell function, which is mediated via IL-10 and dependent on DC-B-cell contact. Considering the importance of B-cell-DC contact, we determined how $B$ cells affect the activation of mature NOD.PI2tg BM-DCs, in the absence of T cells. We found that all $B$ cells significantly increased the survival of DCs in cocultures (Supplementary Fig. 5a); however, B cells from protected NOD mice were more effective at enhancing DC survival compared with B cells from diabetic NOD mice (Supplementary Fig. 5a). In the protected NOD mouse cultures, B-cell contact was required for complete DC deactivation, as we found that MHC I, CD80, CD86, and CD69 expression levels were all significantly downregulated on mature BM-DCs cultured in contact (Cont) with LPS-stimulated B cells compared with those cultured with unstimulated B cells (Fig. 6a, b). B cells from protected NOD mice, when not in direct contact with DCs (Trans), were less effective in deactivating BM-DCs, with reduced downregulation of CD86 and CD69 expression (Fig. 6a, b). Our data suggest that $B$ cells from protected mice suppress MHC I and CD80 expression on BM-DCs via soluble factors, whereas the suppression of CD86 and CD69 expression is mediated via contact-dependent mechanisms.

When investigating $B$ cells from diabetic NOD mice, $B_{\text {LPS }}$ also downregulated the expression of all measured activation markers on DCs if cultured with direct contact (Cont) (Fig. 6a, b). In contrast, when the same B cells were separated (Trans) from DCs, we observed minimal effects on mature BM-DCs. Interestingly, supernatants taken from B cells that had been stimulated in culture with the various stimuli (Sup), had no effect on the activation of BM-DCs compared with DC controls (dotted line, Fig. $6 a, b)$. However, in transwell cultures containing $B_{\text {LPS }}$ from protected mice, MHC I, CD80, and CD69 expression on the BM-DCs was still significantly reduced compared with the expression levels produced by exposure to $B_{\text {LPS }}$ supernatants, but this effect was not observed in the diabetic B-cell transwell cultures (Fig. 6a, b). This finding suggests that $B$ cells from protected mice can respond to BM-DCs and in turn deactivate the 
a

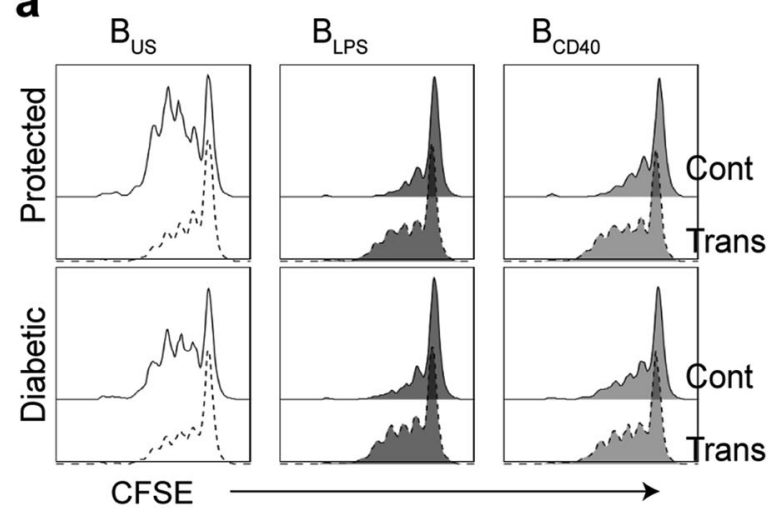

b

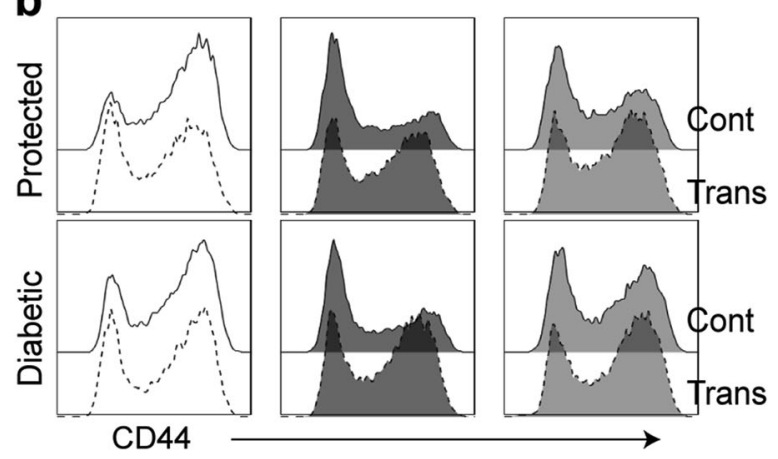

C

Protected

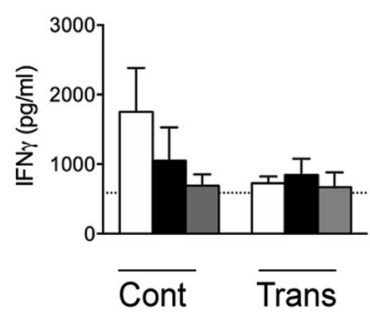

d

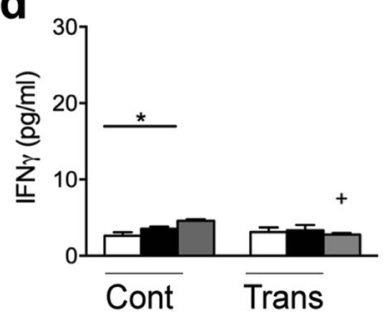

Diabetic

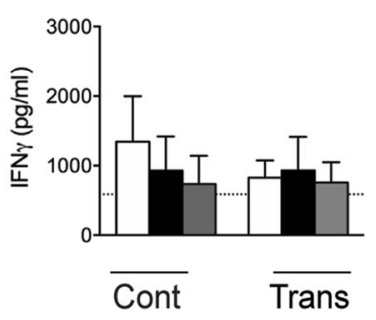

Protected
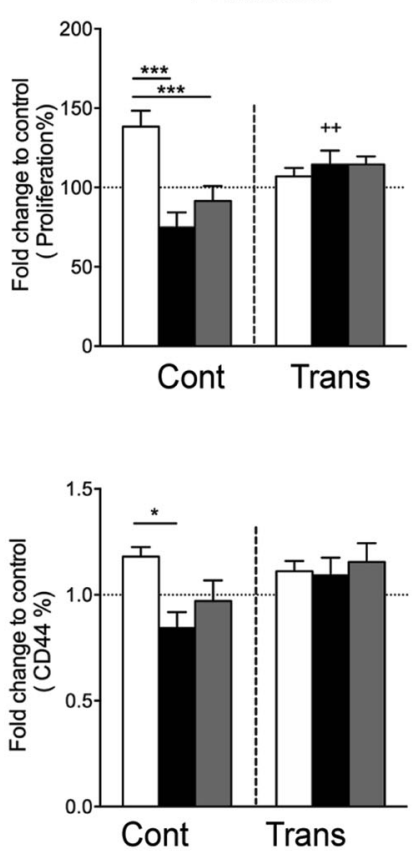

$\mathrm{B}_{\text {US }}$

$\mathbf{e}$

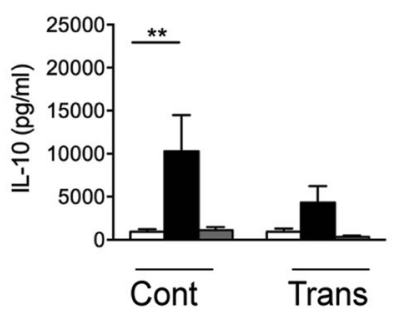

f

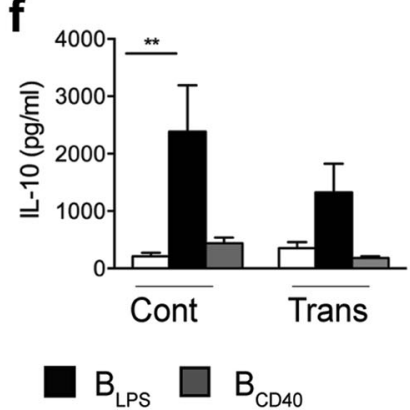

$$
\mathrm{B}_{\text {us }} \square \mathrm{B}_{\text {LPS }} \square \mathrm{B}_{\mathrm{CD} 40}
$$

Diabetic
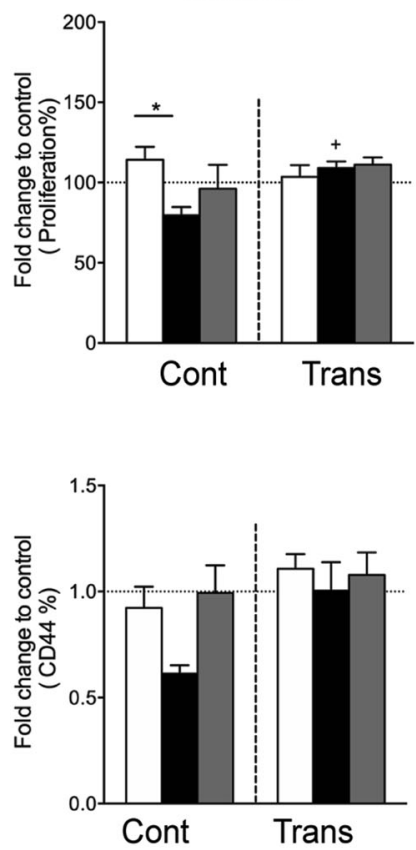

$\mathrm{B}_{\mathrm{LPS}} \square \mathrm{B}_{\mathrm{CD} 40}$

Diabetic
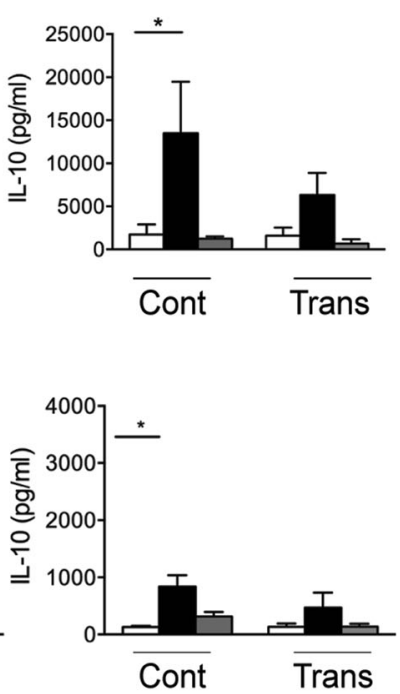

Fig. 4 B-cell-DC-cell contact suppresses CD8 T-cell proliferation and activation. Activated NOD.PI2 ${ }^{\text {tg }}$ BM-DCs and unstimulated (Bus) or LPS $\left(B_{\text {LPS }}\right)$ or anti-CD40-stimulated B cells $\left(B_{a C D 40}\right)$ from protected or diabetic NOD mice were cultured with $G 9 C \alpha^{-1-} C D 8$ T cells $(a-c$, e) or without $\mathrm{G} 9 \mathrm{Co}^{-1-} \mathrm{CD} 8 \mathrm{~T}$ cells $(\mathbf{d}, \mathbf{f})$ for 3 days before analyses. The B cells were cultured either in contact (Cont) or separated from the BM-DCs ${ }^{+/-} \mathrm{CD} 8$ T cells in transwells (Trans). a, b Flow cytometric analyses of CD8 T cells. Representative plots (left) and summary graphs (right) for a CFSE dilution illustrating proliferation and b CD44 surface expression. Data were normalized to DC + CD8 alone controls (dotted line). $\mathbf{c}$, e Supernatants from protected and diabetic NOD DC-B-cell-CD8 T-cell cocultures evaluated for $\mathbf{c}$ IFN $\gamma$ and e IL-10 levels. d, $\mathbf{f}$ Supernatants from protected and diabetic NOD DC-B-cell cocultures evaluated for $\mathbf{d}$ IFN $\gamma$ and $\mathbf{f} \mathrm{IL}-10$. The data are shown as the mean $\pm \mathrm{SEM}$. ${ }^{*} P<0.05,{ }^{* *} P<0.01$, and ${ }^{* * *} P<0.001$ versus $B_{\text {Us }}$ (two-way ANOVA); ${ }^{+} P<0.05$ and ${ }^{++} P<0.01$, Cont versus Trans (two-way ANOVA). Data represent at least three independent experiments 

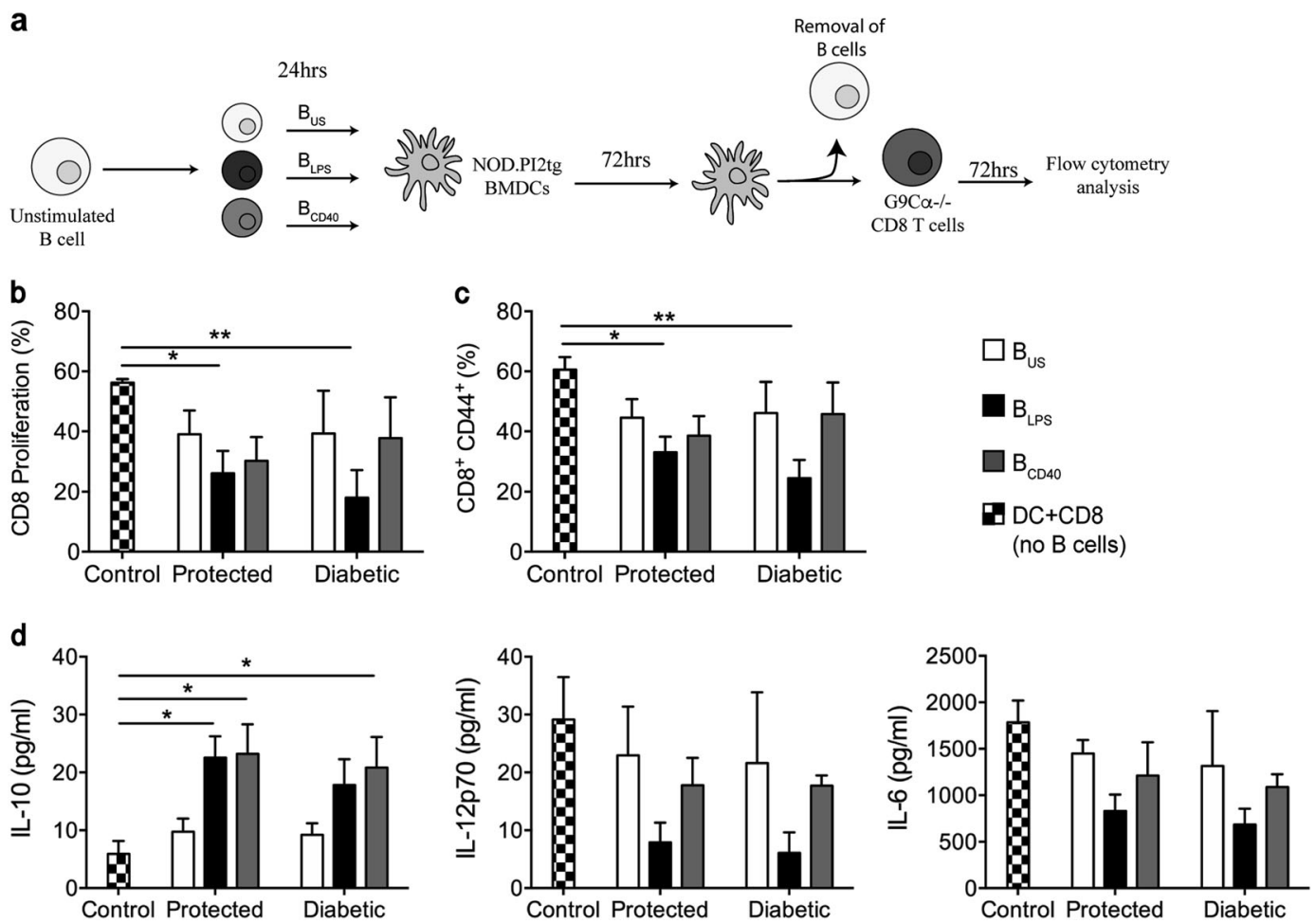

Fig. $5 \quad B_{L P S}$ have lasting tolerogenic effects on mature BM-DCs. Activated NOD.PI2 ${ }^{\text {tg }} B M-D C s$ and unstimulated ( $\left.B_{U S}\right)$ or LPS (B $\left.B_{L P S}\right)$ or antiCD40-stimulated $B$ cells $\left(B_{a C D 40}\right)$ from protected or diabetic NOD mice were cocultured for 3 days before being removed, and the remaining NOD.PI2 ${ }^{\text {tg }}$ BM-DCs were cultured with $\mathrm{G} 9 \mathrm{C}^{-/-} \mathrm{CD} 8 \mathrm{~T}$ cells for 3 days before being assessed by flow cytometry. a Experimental setup. $\mathbf{b}$ CFSE dilution illustrating CD8 T-cell proliferation. c CD44 surface expression on CD8 T cells. The CD8 T cells were gated on live $\mathrm{CD} 11 \mathrm{~b}^{-} \mathrm{CD} 11 \mathrm{c}^{-} \mathrm{CD} 19^{-} \mathrm{CD} 8^{+}$cells. d Cytokine analysis of supernatants for IL-10 (left), IL-12p70 (middle), and IL-6 (right). Control (checkered bar) represents DCs not cultured with B cells prior to the addition of CD8 T cells. The data are shown as the mean \pm SEM. ${ }^{*} P<0.05$ and ${ }^{* *} P<$ 0.01, two-way ANOVA. Data represent three independent experiments

BM-DCs. Conversely, in B cells from diabetic mice, this function is absent. Therefore, $B$ cells from diabetic mice are less effective in mediating the deactivation of mature BM-DCs, but can regain some of this functionality if stimulated via TLR4 and cultured in direct contact with BM-DCs.

Notably, B cells from protected or diabetic NOD mice stimulated via TLR4 or anti-CD40 also significantly downregulated MHC II expression (Supplementary Fig. 5b, c), independent of cell contact. Collectively, these results reveal that the "deactivation" of mature conventional BM-DCs by B cells requires both direct contact and soluble mediators; MHC I, MHC II, and CD80 are more affected by soluble factors (most likely cytokines), whereas CD86 and CD69 are more dependent on direct cell contact. Furthermore, $B$ cells that receive signaling via TLR4 relatively efficiently "deactivate" BM-DC, and B cells from protected NOD mice are the most effective.

$B_{\text {LPS }}$ cells induce tolerogenic $C D 45 R^{+} C D 11 c^{\text {low }} B M-D C s$

To determine if LPS-stimulated $B$ cells not only deactivate mature BM-DCs but also induce a tolerogenic DC population, we cultured $B$ cells and NOD.PI2tg BM-DCs and evaluated different tolerogenic surface markers (Fig. 7). We demonstrated that DCs cocultured with $B_{\text {LPS }}$ cells from protected or diabetic NOD mice exhibited significant induction of a subset of CD45RB ${ }^{+}$DCs compared with DCs cultured alone or with $B_{\text {US }}$ (Fig. 7a). However, $B_{\text {LPS }}$ from protected NOD mice induced significantly more $\mathrm{CD} 45 \mathrm{RB}^{+} \mathrm{DCs}$ compared with $\mathrm{B}_{\mathrm{LPS}}$ from diabetic NOD mice $(p<0.05)$. Interestingly, $B$ cells from diabetic mice stimulated with anti-CD40 ( $\left.B_{C D 40}\right)$ also induced a $C D 45 \mathrm{RB}^{+}$population. Furthermore, the induction of $\mathrm{CD}_{45 \mathrm{RB}^{+}} \mathrm{DCs}$ was dependent on B-cell production of IL-10, as neither $B_{L P S}$ nor $B_{C D 40}$ from IL-10KO mice induced CD45RB expression on BM-DCs (Fig. 7a). Similarly, the addition of $B$ cells from $\mathrm{B} 6^{\mathrm{G} 7}$ mice, which have a reduced number of IL-10-producing $B$ cells, had no effect on the induction of CD45RB expression on BM-DCs (data not shown).

This population of BM-DCs with CD45RB expression, a phenotype associated with regulatory DCs, had lower expression of $\mathrm{CD} 11 \mathrm{C}$ compared with the $\mathrm{CD}_{45 \mathrm{RB}^{-}} \mathrm{BM}-\mathrm{DC}$ population (Fig. 7b, c). ${ }^{23,24}$ Furthermore, significantly fewer $\mathrm{CD}_{45 \mathrm{RB}}{ }^{+} \mathrm{BM}-\mathrm{DCs}$ had $\mathrm{CD} 80$ or CD86 expression (Fig. 7b, c) compared with CD45RB ${ }^{-}$BM-DCs. No difference in the $\mathrm{CD}^{2} \mathrm{RRB}^{+}$phenotype was observed between protected and diabetic cultures; therefore, the overall phenotype is shown (Fig. 7b, c). We also investigated the expression of PD-L1 and ILT3 on BM-DCs with or without B-cell contact and did not find any obvious differences (data not shown), indicating that the tolerogenic DCs induced by $B$ cells in this study did not function through the inhibitory markers PD-L1 and ILT3. ${ }^{25,26}$

\section{DISCUSSION}

We have several novel findings in this study. First, we report that B cells from NOD mice that are naturally protected from diabetes have an increased frequency of IL-10-expressing B cells, while $B$ cells from nonprotected diabetic NOD mice show an altered IL-10 profile (Fig. 8, parts 1, 2). Second, we show that TLR4-activated B cells amplify their IL-10 production in response to contact with mature BM-DCs, inducing a tolerogenic DC state that can suppress pathogenic CD8 T cells (Fig. 8, part 3). Third, B cells from diabetic NOD mice have a diminished response to BM-DCs (Fig. 8, part 4); however, they regain IL-10 production and the function of suppressing insulin-specific CD8 T cells in the presence of proinflammatory cytokines and TLR stimulation (Fig. 8, part 5). Last, we show that B cells deactivate mature BM-DCs via both soluble mediators and cell contact-dependent mechanisms. 
a
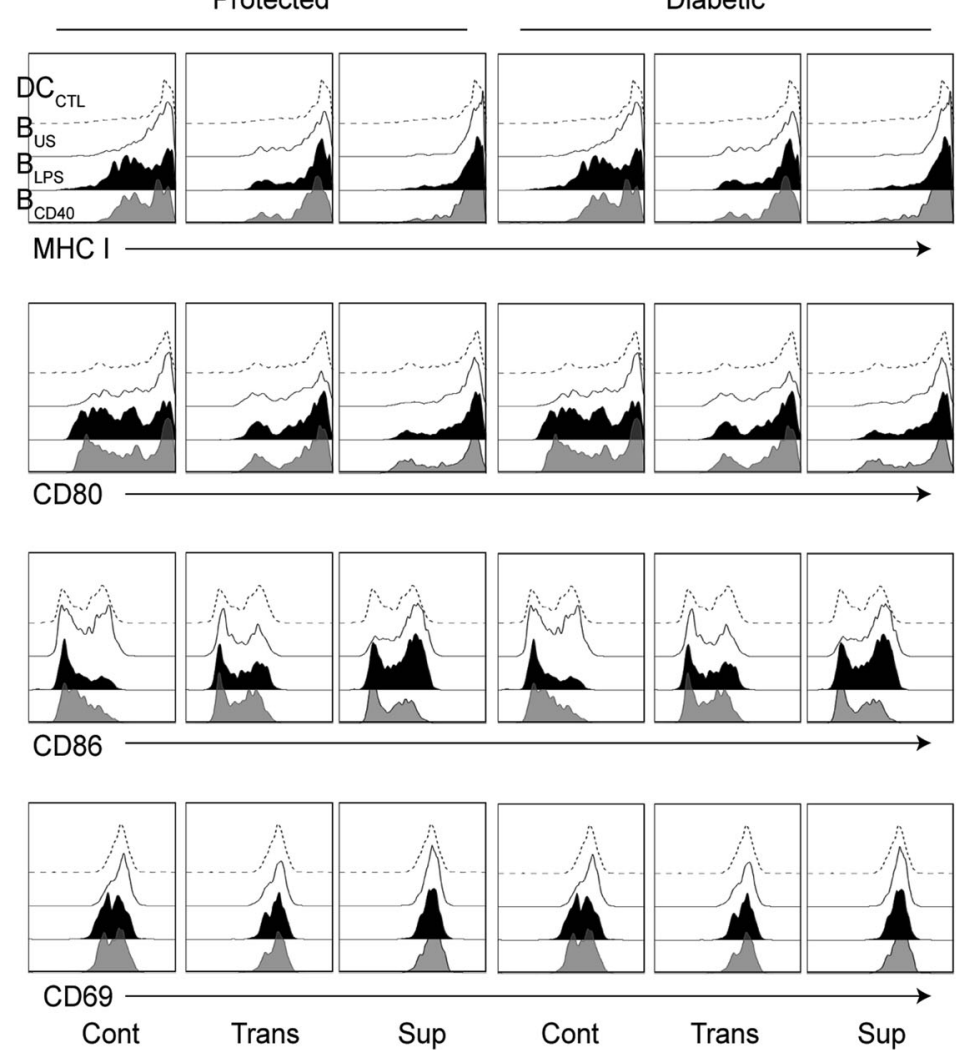

b
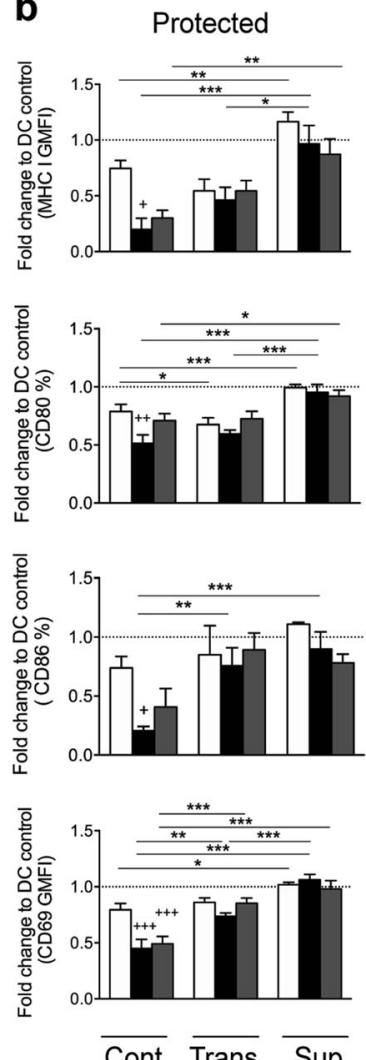

$\overline{\text { Cont }} \overline{\text { Trans }} \overline{\text { Sup }}$
Diabetic
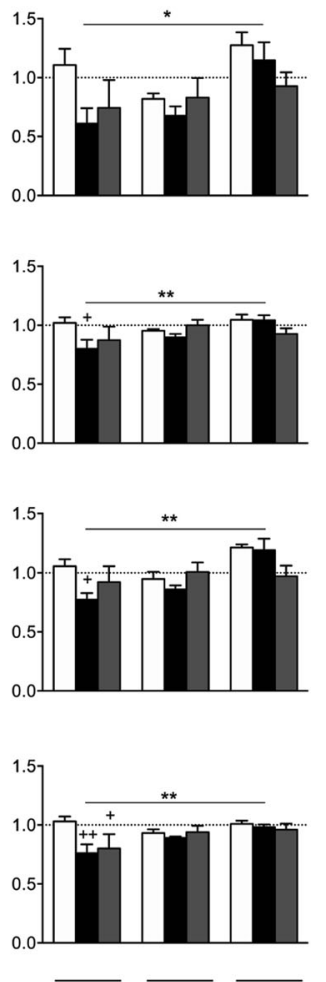

$\overline{\text { Cont }} \overline{\text { Trans }} \overline{\text { Sup }}$

$\mathrm{B}_{\mathrm{LPS}} \square \mathrm{B}_{\mathrm{CD} 40}$

Fig. 6 B cells modulate mature DC activation. Mature NOD.PI2 ${ }^{\text {tg }}$ BM-DCs and unstimulated (Bus) or LPS- (BPS) or anti-CD40-stimulated B cells $\left(\mathrm{B}_{\mathrm{aCD} 40}\right)$ from protected or diabetic NOD mice were cocultured for 3 days before analysis. $\mathbf{a}$, $\mathbf{b}$ DC-B-cell cocultures cultured either in contact (Cont) or in a transwell plate (Trans) or BM-DCs treated with supernatants from B cells that were either left unstimulated or treated with different stimuli (24 h prior to coculture) (Sup) and mature NOD.PI2 ${ }^{\text {tg }}$ DCs analyzed for various surface markers. a Representative plots and $\mathbf{b}$ combined graphical data. The dotted line represents the DC control (no B cells). All data were normalized to DC control cultures. ${ }^{*} P<0.05, * * P$ $<0.01$, and ${ }^{* * *} P<0.001$, cont versus trans versus sup (two-way ANOVA); ${ }^{+} P<0.05,{ }^{++} P<0.01$, and ${ }^{+++} P<0.001$ versus $B_{\text {Us }}$ (two-way ANOVA). The data are shown as the mean \pm SEM. Data represent at least three independent experiments

IL-10-producing B cells (B10) restrain inflammatory responses, and it has been widely documented that B10 cells can negatively regulate autoimmune diseases. Furthermore, it is implied that IL10-producing B cells are lost in patients with type 1 diabetes compared with healthy control individuals. ${ }^{13}$ Notably, B10 cells can be induced through both adaptive immune (anti-CD40) signaling and innate TLR signaling, ${ }^{16}$ which are regulated via different mechanisms, ${ }^{2,27,28}$ under various inflammatory conditions. Our results reveal a loss of cytoplasmic IL-10 in nonprotected diabetic NOD mice, specifically upon stimulation with CD40. In line with this finding, compared with healthy control subjects, patients with systemic lupus erythematosus (SLE) have impaired $\mathrm{CD} 19^{+} \mathrm{CD} 24^{\mathrm{hi}} \mathrm{CD} 38^{\text {hi }}$ Breg populations that are refractory to CD40 stimulation. ${ }^{29}$ This impairment is associated with a lack of STAT-3 phosphorylation after CD40 engagement and the expression of CD40 was not altered. ${ }^{29}$ There was no difference in CD40 expression on splenic B cells between protected and diabetic mice in our study (data not shown) or in the studies of others. $^{13}$ The lack of an IL-10 response following anti-CD40 stimulation could be due to other factors. These other factors include retention of IL-10 by B cells from protected mice, trafficking out of the spleen of $B$ cells that respond to CD40 stimulation, and destruction of $B$ cells in vitro after stimulation (however, no difference was observed in B-cell viability) or in vivo by other cell types, such as $\mathrm{FasL}^{+} \mathrm{CD}^{+} \mathrm{B}$ cells. ${ }^{30}$ Altogether, our current results support the concept that there is a strong association between CD40 stimulation and the immune regulation of autoimmune disease., ${ }^{4,31}$

Importantly, our study shows, for the first time, that the impaired IL-10 response by B cells in diabetic NOD mice can be restored upon activation via TLR4 or TLR9 signaling. Under these circumstances, the secretion of IL-10 by B cells in diabetic NOD mice was increased compared with that of $B$ cells from protected mice stimulated with CPG, or to a lesser extent, LPS. Considering that we found that $B$ cells from diabetic mice were more activated, it is possible that these cells are primed to secrete cytokines more rapidly. Although others have reported that LPS-stimulated B cells from very young NOD mice exert regulatory effects on type 1 diabetes via secreted TGF $\beta{ }^{2}$ we did not detect any TGF $\beta$ secretion in our assays (data not shown). This disparity may be due to the differences in the ages of the mice studied.

In this study, we demonstrate that NOD B cells can deactivate mature BM-DCs via both soluble mediators and cell contactdependent mechanisms. TLR4-stimulated B cells produced more IL-10 and exhibited a stronger capacity to deactivate BM-DCs than unstimulated B cells; specifically, the expression of MHC I, MHC II, and CD80 on BM-DCs was clearly reduced. Furthermore, the expression of CD86 and CD69 was downregulated by direct Bcell-DC contact. Importantly, B cells from diabetic NOD mice stimulated via anti-CD40 were not as effective in deactivating DCs 
a

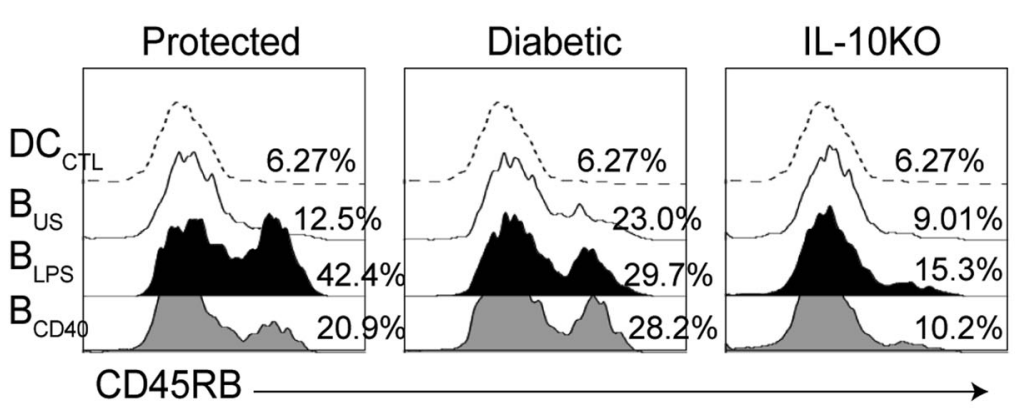

b

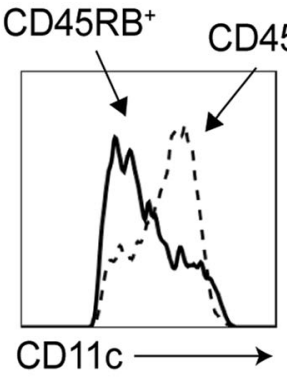

\section{CD45RB-}

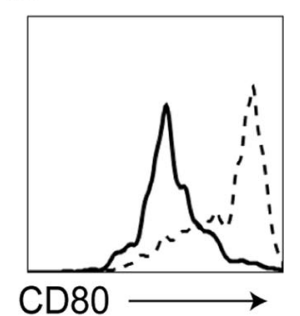

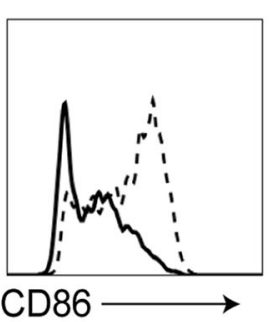
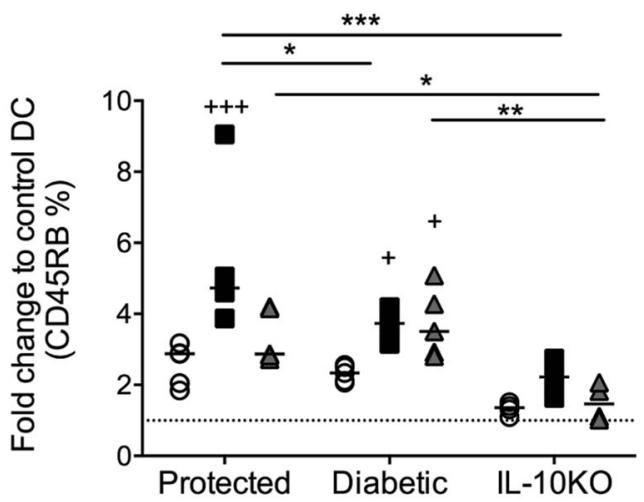

$\mathrm{OB}_{\text {us }} \square \mathrm{B}_{\text {LPS }}$

$\mathrm{B}_{\mathrm{CD} 40}$

C
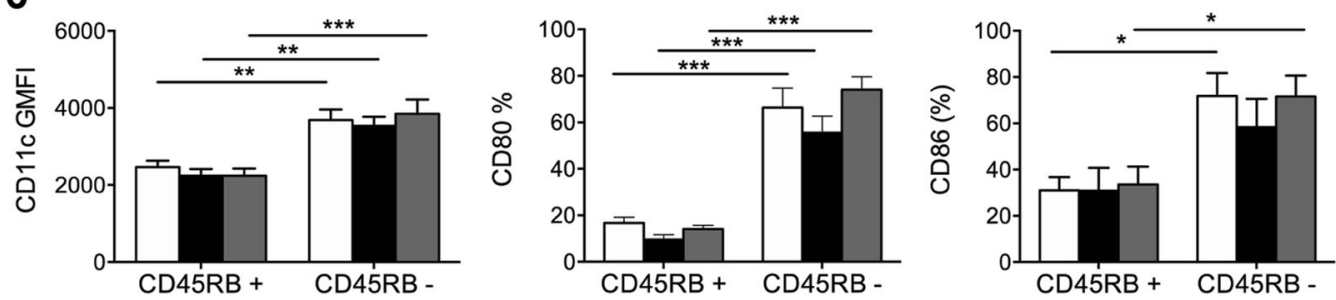

Fig. $7 \mathrm{~B}_{\mathrm{LPS}}$ induce a CD45RB ${ }^{+} \mathrm{CD} 11 \mathrm{c}^{\mathrm{lo}}$ tolerogenic DC population. Activated NOD.PI2 ${ }^{\text {tg }} \mathrm{BM}-\mathrm{DCs}$ and unstimulated (Bus, white circle) or $\mathrm{LPS}$ ( $B_{L P S}$, black square) or anti-CD40-stimulated $B$ cells ( $B_{a C D} 40$, gray triangle) from protected or diabetic or IL-10KO NOD mice were cocultured for 3 days before analysis. a Representative plots (left) and a summary graph (right) of CD45RB ${ }^{+} \mathrm{DC}$ (gated on live $\mathrm{CD} 11 \mathrm{c}^{+} \mathrm{CD} 11 \mathrm{~b}^{+}$) populations. The dotted line represents DCs alone (control DCs). The horizontal line represents the median value. b Representative flow plots for CD11C, CD80, and CD86 expression in CD45RB (solid line) and CD45RB (dashed line) populations. c CD11c, CD80, and CD86 summary graphs for $\mathrm{CD}_{45 R B}{ }^{+}$and $\mathrm{CD}_{45 \mathrm{RB}^{-}}$populations pooled from protected or diabetic NOD mice. The data are shown as the mean $\pm \mathrm{SEM}$. The data shown represent three independent experiments. ${ }^{*} P<0.05$, ${ }^{* *} P<0.01$, and ${ }^{* * *} P<0.001$; two-way ANOVA

as B cells from protected NOD mice. This could be, in part, due to the increased secretion of IFNY upon B-cell-DC contact in diabetic NOD mice. In addition, we found that BM-DCs exposed to LPSstimulated $B$ cells produced reduced amounts of IL-12p70 and IL- 6 but an increased amount of IL-10, in line with the B-cell-DC interactions noted previously. ${ }^{32} \mathrm{~B}$ cells activated to produce IL-10 by Leishmania major infection induce suppression of IL-12 production by DCs. ${ }^{33}$ Similarly, CpG-activated neonatal B cells are able to suppress IL-12 production by neonatal dendritic cells. ${ }^{34}$ Direct B-cell-DC interactions have been shown using B-celldeficient $\left(\mu \mathrm{MT}^{-1}\right)$ mice, whose DCs produce higher levels of IL12 p70 than those from wild-type animals. ${ }^{35}$ Furthermore, it is known that DCs cultured with IL-10 can shift from a Th1 pathway by reducing IL-12 secretion, ${ }^{21}$ and IL-10 can also affect DC antigen presentation. $^{36}$ It is conceivable that the reduction in MHC II expression on BM-DCs induced by IL-10-producing B cells in our study could impact antigen presentation by DCs to CD4 T cells, leading to suboptimal CD4 T-cell activation.

It is clear that TLR4-activated NOD B cells operate directly on BM-DCs to inhibit CD8 T-cell activation. We found that B-cell-DC contact also amplified B-cell secretion of IL-10, which was exaggerated in the presence of IFNY-producing CD8 T cells. Our finding is consistent with that of a previous study suggesting that inflammatory cytokines can increase IL-10 production by Breg cells. ${ }^{37}$ However, we also found that IL-10 alone was not sufficient to inhibit BM-DC-induced CD8 T-cell proliferation, suggesting a contact-dependent change in BM-DCs upon initial engagement with $B$ cells. Furthermore, whether this initial contact-dependent change is reciprocal and whether CD45RB ${ }^{\text {hi }} \mathrm{CD} 11 \mathrm{c}^{\text {low }} \mathrm{DCs}$ have any reverse effects on $B$ cells are not yet known.

In this study, we also demonstrated IL-10-dependent induction

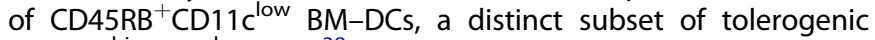
CD45RB ${ }^{\text {hi }} C D 11 c^{\text {low }} D C s,{ }^{38}$ which were induced most efficiently with LPS-stimulated B cells from protected NOD mice. A previous study suggests that a similar tolerogenic DC population produces $\mathrm{IL}-27$ and promotes T-cell tolerance via IL- $10 .^{24}$ Interestingly, this population can be induced with galectin- $1,{ }^{24}$ which has recently been described to be required for regulatory $B$ cell functions. ${ }^{39}$ Whether this mechanism is involved in the induction of the

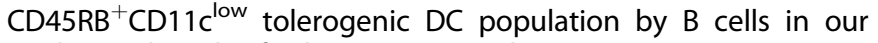
study needs to be further investigated.

Our results are in line with findings on human B-cell-DC interactions, showing that human $B$ cells influence the differentiation of DCs. ${ }^{40-42}$ B cells activated by CD40 and TLR9 can also restrict 


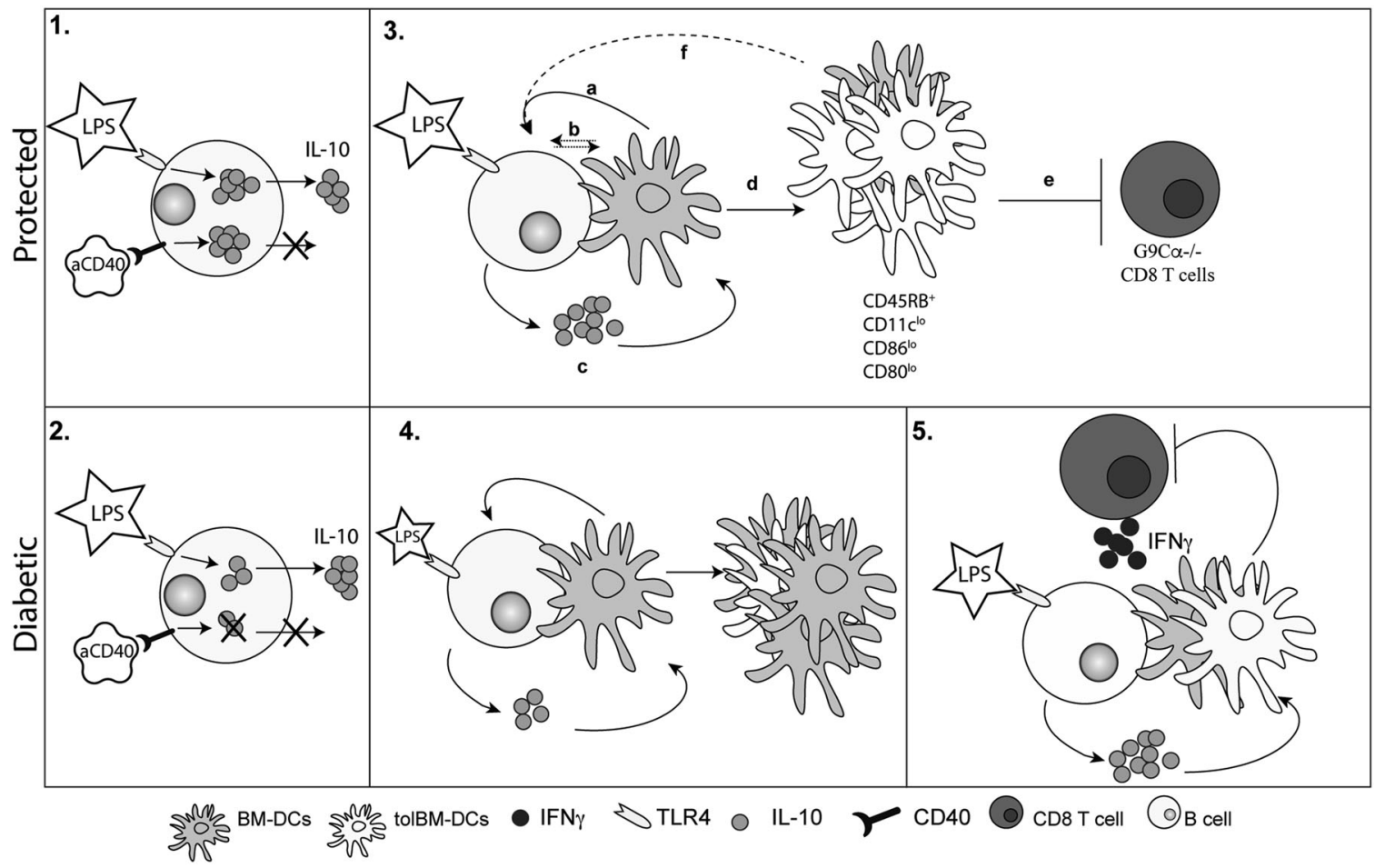

Fig. 8 Schematic summary of B-cell-directed CD8 T-cell suppression in the context of type 1 diabetes. 1. B cells from protected NOD mice stimulated via TLR4 or anti-CD40 express substantial levels of intracellular IL-10, but only TLR4 stimulation induces IL-10 secretion. 2. B cells from diabetic NOD mice express reduced levels of intracellular IL-10 when stimulated via TLR4 and lack IL-10 expression in response to antiCD40 stimulation but can secrete IL-10 in response to TLR4 stimulation. 3. a BM-DCs engage with TLR4-activated B cells from protected NOD mice. b Upon initial engagement, a possible reciprocal contact-mediated process induces altered BM-DC to generate an IL-10 feedback loop and tolBM-DC induction by IL-10 (dotted arrows). c B cell-BM-DC contact augments IL-10 production by B cells, creating a feedback loop as shown by the solid black arrows. d B cell-BM-DC contact and IL-10 secretion induce a deactivated tolerogenic BM-DC population (tolBM-DCs). e Tolerogenic deactivated BM-DCs suppress pathogenic CD8 T cells. $f$ The effect of tolBM-DCs on B cells after induction is still unknown (dashed arrow). 4. B cells from diabetic NOD mice have a diminished response to contact with BM-DCs, which results in fewer deactivated tolBM-DCs. 5. In the presence of CD8 T cells and proinflammatory cytokines, i.e., IFN $\gamma$, LPS-stimulated B cells from diabetic NOD mice in contact with BM-DCs amplify their IL-10 response and induce CD8 T-cell suppression as effectively as B cells from protected NOD mice

monocytes from developing into mature DCs and reduce the expression of activation molecules and production of cytokines by DCs. ${ }^{40}$ Similarly, B cells activated via BCR signaling can induce DC maturation, which then drives the differentiation of CD4 T cells into Th2 cells. ${ }^{42}$ Again, this maturation is dependent on B-cell-DC contact and B-cell factors such as BAFFR (B-cell-activating factor receptor), TACI (transmembrane and calcium-modulating cyclophilin ligand interactor), and CD69. ${ }^{42}$ It is clear that there is important cross-talk between $B$ cells and DCs, and this is dependent on which signals B cells receive. ${ }^{41}$ Our results suggest that the cross-talk between B cells and DCs is mutually modulated and both cell contact dependent and cell contact independent.

In summary, we have found that B cells play a novel role in the natural protection of NOD mice from diabetes. B cells from protected NOD mice produce high levels of IL-10 and suppress the activation of BM-DCs, which control pathogenic CD8 T cells. In contrast, B cells from nonprotected diabetic NOD mice have reduced IL-10 expression, especially upon activation with CD40, and weak suppressive function. Interestingly and importantly, if $B$ cells from nonprotected diabetic mice are stimulated through innate immune signaling pathways, in particular the TLR4 (LPS) pathway, these B cells have the capacity to produce IL-10, and the immunosuppressive function is restored. This alteration of the suppressive B-cell function under innate immune activation or inflammatory conditions may contribute to the dysregulation or abnormalities in DC populations found in individuals with type 1 diabetes. $^{43}$ Thus, our study may point to a possible therapeutic target for future investigation.

\section{METHODS}

Mice

NOD/Caj mice, originally from Yale University, were bred in-house at Cardiff University. G9Ca ${ }^{-/-}$NOD mice were bred in-house at Cardiff University as previously described. ${ }^{20}$ NOD TLR4 $^{-/-}$mice were bred in-house at Yale University. NOD.PI2 ${ }^{\text {tg }}$ mice, which have transgenic overexpression of PI2 driven by the MHC class II promotor, were kindly provided by Prof. L. Harrison and Dr. A. Lew. $\mathrm{B}^{\mathrm{g}^{7}}$ mice were bred in-house at Cardiff University. NOD.129P2 (Cg)-II10tm1Cgn/DvsJ (IL-10KO) mice were bred in-house at Yale University. Mice were maintained at Cardiff or Yale University in specific pathogen-free isolators or scantainers. All animals received water and food ad libitum and were housed with a 12h dark/light cycle. Animal experiments were conducted in accordance with the United Kingdom Animals (Scientific Procedures) Act, 1986 and associated guidelines.

\section{Diabetes incidence}

Mice were monitored weekly for glycosuria (Bayer Diastix) beginning at 12 weeks of age and diagnosed as diabetic when blood glucose levels were $>13.9 \mathrm{mmol} / \mathrm{L}$. NOD mice that were 35 weeks of age or older, had never tested positive for glycosuria, and had blood glucose levels $<13.9 \mathrm{mmol} / \mathrm{L}$ were considered to be protected from diabetes, as the incidence of diabetes after this age is very low.

\section{Reagents}

In vivo Mab anti-mouse IL-10R (CD210), In vivo Mab rat IgG1 isotype control, anti-CD3 (clone 2C11), and anti-CD28 (clone 37.51) 
antibodies were all purchased from Bioexcell. Recombinant IL-10 was purchased from Miltenyi Biotec.

\section{Cell preparation}

Bone marrow (BM-DCs) cells were flushed out from the hind leg bones (femur and tibia), cultured with granulocyte macrophage colony-stimulating factor (GM-CSF) at $1.5 \mathrm{ng} / \mathrm{ml}$, and stimulated overnight with LPS (Sigma) at $1 \mu \mathrm{g} / \mathrm{ml}$ before the coculture system was set up. Whole splenocytes or freshly isolated splenic B cells, which were selected by using a $B$ cell isolation kit (Miltenyi), were seeded at either $1 \times 10^{6} / \mathrm{ml}$ in a 24-well plate (for phenotyping) or $5 \times 10^{6}$ cells $/ \mathrm{ml}$ in a six-well plate (for cocultures) and either left unstimulated or stimulated with $5 \mu \mathrm{g} / \mathrm{ml}$ lipopolysaccharide (LPS), $5 \mu \mathrm{g} / \mathrm{ml}$ anti-CD40 (Bioexcell), or $0.5 \mu \mathrm{g} / \mathrm{ml} \mathrm{CPG} \mathrm{(Eurofins} \mathrm{MWG)} \mathrm{for}$ $24 \mathrm{~h}$ before harvesting for further assays. CD8 T cells were negatively selected with a CD8 T cell isolation kit (Miltenyi). The purity following all cell-sorting processes was $>95 \%$.

\section{B-cell-DC cocultures}

NOD.PI2 ${ }^{\text {tg }}$ BM-DCs that were stimulated with LPS were cultured either alone or with B cells, unstimulated, or stimulated with various stimuli, at a ratio of 1:3 (DCs:B cells) in a 24-well plate for 3 days. For transwell experiments, $B$ cells and DCs were separated by a $0.4-\mu \mathrm{M}$ membrane (Corning), with the DCs placed in the bottom chamber and unstimulated/stimulated NOD B cells placed in the top chamber. For DCs cultured with various NOD B-cell supernatants, the supernatants were centrifuged to ensure cell removal before addition to $4 \times 10^{5}$ DCs ( $1 \mathrm{ml}$ per well). After 3 days, assays were analyzed by flow cytometry.

B-cell-DC-CD8 T-cell cocultures

NOD.PI2 ${ }^{\text {tg }}$ BM-DCs stimulated with LPS were cultured with freshly isolated $\mathrm{G} \mathrm{Ca}^{-1-} \mathrm{CD} 8 \mathrm{~T}$ cells with or without unstimulated or stimulated NOD or TLR4 ${ }^{-/-}$B cells at a ratio of 1:2:7 (CD8 T cells: DCs:B cells) for 3 days. The $\mathrm{G} \mathrm{Ca}^{-1-} \mathrm{CD} 8 \mathrm{~T}$ cells were labeled with $0.5 \mu \mathrm{mol} / \mathrm{L}$ CFDA-SE (CFSE) (Invitrogen) before the cocultures were set up. For transwell experiments (Corning), 0.4- $\mu \mathrm{M}$ membranes separated B cells from BM-DCs and CD8 T cells, with the unstimulated/stimulated $B$ cells placed in the top chamber and the BM-DCs and CD8 T cells mixed in the bottom. For IL-10R blocking experiments, $10 \mu \mathrm{g} / \mathrm{ml}$ anti-IL-10R antibody or isotype control was added for the 3 -day culture. NOD.PI2 ${ }^{\text {tg }}$ BM-DCs $(4 \times$ $10^{5}$ ) were also cultured with recombinant IL-10 (Miltenyi Biotech) alone, at increasing concentrations. Plate-bound anti-CD3/CD28 antibodies $(0.1 \mu \mathrm{g} / \mathrm{ml}$ anti-CD3 antibody, $1 \mu \mathrm{g} / \mathrm{ml}$ anti-CD28 antibody) were used to coat a 48 -well plate overnight at $4{ }^{\circ} \mathrm{C}$. The plate was washed with PBS before plating $\mathrm{G} 9 \mathrm{Ca}^{-/-} \mathrm{CD} 8 \mathrm{~T}$ cells labeled with CFDA-SE, and the cells were cultured with or without NOD B cells treated with different stimuli. After 3 days, the assays were analyzed by flow cytometry.

\section{Flow cytometry}

Single-cell suspensions were incubated with TruStain (antimouse CD16/32 antibodies [BioLegend]) for $10 \mathrm{~min}$ at $4{ }^{\circ} \mathrm{C}$, followed by incubation with fluorochrome-conjugated $\mathrm{mAbs}$ against cell surface markers for $30 \mathrm{~min}$ at $4{ }^{\circ} \mathrm{C}$. Multiparameter flow cytometry was carried out by using the following mAbs: CD8 PE594 (53-6.7), CD19 AF700 (6D5), CD11b APC or BV421 (M1/70), CD11C Pe-Cy7 (N418), MHC I PE (SF1-1.1), MHC II FITC (10-3-6), CD80 BV650 (16-10A1), CD86 Pe-Cy7 or AF700 (PO3), CD44 BV711 (IM7), PD-L1 APC (10F-962), OX-40L Pe-Cy7 (RM134), CD69 BV510 (H1-2F3), and CD45RB PerCPCy5.5 (C363-16.7) (all from BioLegend). APC-Cy7- (1D3) and PerCPCy5.5-conjugated (N418) anti-CD11c antibodies were purchased from eBioscience, and a BV786-conjugated BAFFR antibody (7H22-E16) was purchased from BD Biosciences. Cells were also stained with live/dead exclusion 605 (Invitrogen) or 780 (eBioscience). For intracellular cytokine analysis, splenocytes were either left unstimulated or stimulated for $24 \mathrm{~h}$. Three hours before antibody staining, PMA $(50 \mathrm{ng} / \mathrm{ml})$, ionomycin $(500 \mathrm{ng} / \mathrm{ml})$, and monensin $(3 \mu \mathrm{g} / \mathrm{ml})$ (all from Sigma-Aldrich) were added to the cells. Fc receptors were blocked by using TruStain, and after extracellular staining, the cells were fixed by using a fixation/ permeabilization kit according to the manufacturer's instructions (BD Biosciences) and subsequently stained with antibodies specific for intracellular cytokines or appropriate isotype controls. Cells were acquired on an LSRFortessa (FACSDiva software), and analysis was performed by using FlowJo software (TreeStar).

\section{Cytokine assays}

Supernatants were taken from cell culture assays at the 3-day endpoint to analyze IL-10, IL-12p70, MIP1 $\beta$, IFN $\gamma$, and IL-6. Levels of the cytokines IL-10, IL-12p70, IFN $\gamma$, and IL- 6 were measured by a Meso Scale Discovery (MSD) system, and the MIP1 $\beta$ level was measured by ELISA (R\&D systems). MSD was performed according to the manufacturer's (Meso Scale Diagnostics, LLC) instructions and evaluated by using the MSD Sector Imager 6000 . The MIP1 $\beta$ ELISA was run according to the manufacturer's instructions (R\&D systems).

Statistical analysis

Statistical analysis was performed by using GraphPad Prism (GraphPad Software, San Diego, CA). Significance was determined by one-way ANOVA followed by Dunn's multiple comparison test or two-way ANOVA followed by the Bonferroni posttest for more than two variables, and a Mann-Whitney $U$ test was performed to compare only two variables. Data were considered significant at $p<0.05$.

\section{DATA AVAILABILITY}

The datasets generated or analyzed in this study are available upon reasonable request.

\section{ACKNOWLEDGEMENTS}

This work was funded by the Medical Research Council (UK) (MR/K021141/1 to FSW). LCDR was funded by a studentship from Cnpq (Conselho Nacional de Pesquisa, Brazil, grant \#245609/2012-1).

\section{AUTHOR CONTRIBUTIONS}

J.B., L.W. and F.S.W. designed the experiments and wrote the paper. J.B. performed the experiments and analyzed the data. L.C.daR. and J.D. contributed to experimental procedures. All authors reviewed the paper. F.S.W. conceived the project and is the guarantor of this work.

\section{ADDITIONAL INFORMATION}

The online version of this article (https://doi.org/10.1038/s41423-019-0324-z) contains supplementary material.

Competing interests: The authors declare no competing interests.

\section{REFERENCES}

1. Mizoguchi, A., Mizoguchi, E., Takedatsu, H., Blumberg, R. S. \& Bhan, A. K. Chronic intestinal inflammatory condition generates IL-10-producing regulatory $B$ cell subset characterized by CD1d upregulation. Immunity 16, 219-230 (2002).

2. Tian, J. et al. Lipopolysaccharide-activated B cells down-regulate Th1 immunity and prevent autoimmune diabetes in nonobese diabetic mice. J. Immunol. 167, 1081-1089 (2001).

3. Shen, P. et al. IL-35-producing B cells are critical regulators of immunity during autoimmune and infectious diseases. Nature 507, 366-370 (2014).

4. Fillatreau, S., Sweenie, C. H., McGeachy, M. J., Gray, D. \& Anderton, S. M. B cells regulate autoimmunity by provision of IL-10. Nat. Immunol. 3, 944-950 (2002).

5. Evans, J. G. et al. Novel suppressive function of transitional 2 B cells in experimental arthritis. J. Immunol. 178, 7868-7878 (2007). 
6. Oleinika, K. et al. CD1d-dependent immune suppression mediated by regulatory B cells through modulations of iNKT cells. Nat. Commun. 9, 684 (2018).

7. $\mathrm{Hu}, \mathrm{C}$. Y. et al. Treatment with CD20-specific antibody prevents and reverses autoimmune diabetes in mice. J. Clin. Invest. 117, 3857-3867 (2007).

8. Fiorina, P. et al. Targeting CD22 reprograms B-cells and reverses autoimmune diabetes. Diabetes 57, 3013-3024 (2008).

9. Zekavat, G. et al. In vivo BLyS/BAFF neutralization ameliorates isletdirected autoimmunity in nonobese diabetic mice. J. Immunol. 181, 8133-8144 (2008).

10. Xiao, Z. et al. Inhibition of Fas ligand in NOD mice unmasks a protective role for IL-10 against insulitis development. Am. J. Pathol. 179, 725-732 (2011).

11. Parekh, V. V. et al. B cells activated by lipopolysaccharide, but not by anti-lg and anti-CD40 antibody, induce anergy in CD8+ T cells: role of TGF-beta 1. J. Immunol. 170, 5897-5911 (2003).

12. Hussain, S. \& Delovitch, T. L. Intravenous transfusion of BCR-activated B cells protects NOD mice from type 1 diabetes in an IL-10-dependent manner. $J$. Immunol. 179, 7225-7232 (2007).

13. Kleffel, S. et al. Interleukin-10+ regulatory B cells arise within antigenexperienced $\mathrm{CD} 40+\mathrm{B}$ cells to maintain tolerance to islet autoantigens. Diabetes 64, 158-171 (2015).

14. Anderson, M. S. \& Bluestone, J. A. The NOD mouse: a model of immune dysregulation. Annu. Rev. Immunol. 23, 447-485 (2005).

15. Rosser, E. C. \& Mauri, C. Regulatory B cells: origin, phenotype, and function Immunity 42, 607-612 (2015).

16. Yanaba, K., Bouaziz, J. D., Matsushita, T., Tsubata, T. \& Tedder, T. F. The development and function of regulatory $B$ cells expressing IL-10 (B10 cells) requires antigen receptor diversity and TLR signals. J. Immunol. 182, 7459-7472 (2009).

17. Barr, T. A. et al. B cell depletion therapy ameliorates autoimmune disease through ablation of IL-6-producing B cells. J. Exp. Med. 209, 1001-1010 (2012).

18. Montandon, R. et al. Innate pro-B-cell progenitors protect against type 1 diabetes by regulating autoimmune effector T cells. Proc. Natl Acad. Sci. USA 110, E2199-E2208 (2013).

19. Guleria, I. et al. Mechanisms of PDL1-mediated regulation of autoimmune diabetes. Clin. Immunol. 125, 16-25 (2007).

20. Wong, F. S. et al. Activation of insulin-reactive CD8 T-cells for development of autoimmune diabetes. Diabetes 58, 1156-1164 (2009).

21. De Smedt, T. et al. Effect of interleukin-10 on dendritic cell maturation and function. Eur. J. Immunol. 27, 1229-1235 (1997).

22. Zhou, F. et al. IL-10 deficiency blocks the ability of LPS to regulate expression of tolerance-related molecules on dendritic cells. Eur. J. Immunol. 42, 1449-1458 (2012).

23. Svensson, M., Maroof, A., Ato, M. \& Kaye, P. M. Stromal cells direct local differentiation of regulatory dendritic cells. Immunity 21, 805-816 (2004).

24. Ilarregui, J. M. et al. Tolerogenic signals delivered by dendritic cells to $T$ cells through a galectin-1-driven immunoregulatory circuit involving interleukin 27 and interleukin 10. Nat. Immunol. 10, 981-991 (2009).
25. Chang, C. C. et al. Tolerization of dendritic cells by $T(S)$ cells: the crucial role of inhibitory receptors ILT3 and ILT4. Nat. Immunol. 3, 237-243 (2002).

26. Sumpter, T. L. \& Thomson, A. W. The STATus of PD-L1 (B7-H1) on tolerogenic APCs. Eur. J. Immunol. 41, 286-290 (2011).

27. Wang, R. X. et al. Interleukin-35 induces regulatory B cells that suppress autoimmune disease. Nat. Med. 20, 633-641 (2014).

28. K. Yanaba, et al. A regulatory B cell subset with a unique CD1dhiCD5+ phenotype controls $\mathrm{T}$ cell-dependent inflammatory responses. Immunity $\mathbf{2 8}$ 639-650 (2008).

29. Blair, P. A. et al. CD19(+)CD24(hi)CD38(hi) B cells exhibit regulatory capacity in healthy individuals but are functionally impaired in systemic Lupus Erythematosus patients. Immunity 32, 129-140 (2010).

30. Saxena, A., Yagita, H., Donner, T. W. \& Hamad, A. R. A. Expansion of FasLexpressing CD5. Front. Immunol. 8, 402 (2017).

31. Mauri, C. \& Bosma, A. Immune regulatory function of B cells. Annu. Rev. Immunol. 30, 221-241 (2012)

32. Bayry, J. et al. Modulation of dendritic cell maturation and function by B lymphocytes. J. Immunol. 175, 15-20 (2005).

33. Ronet, C. et al. Regulatory B cells shape the development of Th2 immune responses in BALB/C mice infected with Leishmania major through IL-10 production. J. Immunol. 184, 886-894 (2010).

34. Sun, C. M., Deriaud, E., Leclerc, C. \& Lo-Man, R. Upon TLR9 signaling, CD5+ B cells control the IL-12-dependent Th1-priming capacity of neonatal DCs. Immunity 22, 467-477 (2005).

35. Moulin, V. et al. B lymphocytes regulate dendritic cell (DC) function in vivo: increased interleukin 12 production by DCs from B cell-deficient mice results in T helper cell type 1 deviation. J. Exp. Med. 192, 475-482 (2000).

36. Mittal, S. K. \& Roche, P. A. Suppression of antigen presentation by IL-10. Curr. Opin. Immunol. 34, 22-27 (2015).

37. Rosser, E. C. et al. Regulatory B cells are induced by gut microbiotadriven interleukin-1 $\beta$ and interleukin-6 production. Nat. Med. 20, 1334-1339 (2014)

38. Wakkach, A. et al. Characterization of dendritic cells that induce tolerance and $T$ regulatory 1 cell differentiation in vivo. Immunity 18, 605-617 (2003).

39. Alhabbab, R. et al. Galectin-1 is required for the regulatory function of B cells. Sci. Rep. 8, 2725 (2018).

40. Morva, A. et al. Maturation and function of human dendritic cells are regulated by B lymphocytes. Blood 119, 106-114 (2012).

41. Maddur, M. S., Kaveri, S. V. \& Bayry., J. Regulation of human dendritic cells by B cells depends on the signals they receive. Blood 119, 3863-3864 (2012).

42. Maddur, M. S. et al. Human B cells induce dendritic cell maturation and favour Th2 polarization by inducing OX-40 ligand. Nat. Commun. 5, 4092 (2014).

43. Takahashi, K., Honeyman, M. C. \& Harrison, L. C. Impaired yield, phenotype, and function of monocyte-derived dendritic cells in humans at risk for insulindependent diabetes. J. Immunol. 161, 2629-2635 (1998). 